\title{
Finite families of plane valuations: value semigroup, graded algebra and Poincaré series
}

\author{
Carlos Galindo and Francisco Monserrat
}

\section{Introduction}

The formal definition of valuation was firstly given by the Hungarian mathematician J. Kürschák in 1912 supported with ideas of Hensel. Valuation theory, based on this concept, has been developed by a large number of contributors (some of them distinguished mathematicians as Krull or Zariski) and it has a wide range of applications in different context and research areas as, for instance, algebraic number theory or commutative algebra and its application to algebraic geometry or theory of diophantine equations.

In this paper, we are interested in some applications of valuation theory to algebraic geometry and, particularly, to singularity theory. Valuation theory was one of the main tools used by Zariski when he attempted to give a proof of resolution of singularities for algebraic schemes. In characteristic zero, resolution was proved by Hironaka without using that tool; however there is no general proof for positive characteristic and valuations seem to be suitable algebraic objects for this purpose. Valuations associated with irreducible curve singularities are one of the best known classes of valuations, especially the case corresponding to plane branches where valuations and desingularization process are very related. Germs of plane curves can contain several branches and, for this reason, it is useful to study their corresponding valuations, not only in an independent manner but as a whole $[\mathbf{6}, \mathbf{7}, \mathbf{8}$. Valuations of the fraction field of some 2-dimensional local regular Noetherian ring $R$ centered at $R$, that we call plane valuations, are a very interesting class of valuations which includes the above mentioned family related with branches. These valuations were studied by Zariski and their study was revitalized by the paper [46. Very little is known about valuations in higher dimension.

The aim of this paper is to provide a concise survey of some aspects of the theory of plane valuations, adding some comments upon more general valuations when it is possible. For those valuations, we describe value semigroup, graded algebra and Poincaré series emphasizing on the recent study of the same algebraic objects for finite families of valuations and their relation with the corresponding ones for reduced germs of plane curves.

1991 Mathematics Subject Classification. Primary 14B05, 13A18.

Supported by Spain Ministry of Education MTM2007-64704 and Bancaixa P1-1B2009-03. 
Section 2 of the paper recalls the general notion of valuation and compiles the main known facts with respect to the value group and semigroup of a valuation. We show a new condition, Proposition [2.2, given in [14, that has to do with the number of generators of the value semigroups of Noetherian local domains (see [17] for a more general result). We also give in Proposition 2.4 a numerical condition, called combinatorially finiteness, that those value semigroups satisfy. The graded algebra of a valuation $\nu, \operatorname{gr}_{\nu} R$, is introduced in Section 3. There, we explain how to construct a minimal free resolution of $\operatorname{gr}_{\nu} R$ as a module over a polynomial ring and, in Proposition 3.2. how to compute the dimension of its $i$ th syzygy module. This graded algebra is the main ingredient in the Teissier's idea to prove resolution of singularities. When $\nu$ is plane $\operatorname{gr}_{\nu} R$ is Noetherian, notwithstanding this is not true for higher dimension (see Proposition 3.4). Section 4 is devoted to introduce plane valuations, their main invariants and to classify them by means of an algebraic device that allows us to get parametric equations of the valuations. The introduction and computation of the Poincaré series of plane valuations (with particular attention to the divisorial case) is given in Section 5 .

Finite families of valuations whose value group is that of integer numbers, $\mathbb{Z}$, are considered in Section 6. For them we define the concepts of graded algebra, generating sequence and Poincaré series, explaining that this series is a rational function whenever one considers certain families of valuations which include the divisorial ones in the plane and those associated with a rational surface singularity. Following [19], and also in this section, semigroup of values, generating sequences and Poincaré series for finite families of plane divisorial valuations are explicitly computed. We also add some information given in [10] corresponding to families of any type of plane valuations. Finally, in Section 7 we provide an specific calculation of the Poincaré series of multiplier ideals of a plane divisorial valuation $\nu$, Theorem 7.5. That series gathers information on the multiplier ideals and jumping numbers corresponding to the singularity that $\nu$ encodes and the proof of Theorem 7.5 uses techniques and results involving the family of plane divisorial valuations given by the exceptional divisors appearing in the blowing-up sequence determined by $\nu$.

\section{Valuations}

2.1. Definition and a bit of history. Between 1940 and 1960, Zariski [51, 52 and Abhyankar [2, 3] developed the theory of valuations in the context of the theory of singularities with the aim of proving resolution for algebraic schemes. The concept of valuation is analogue to that of place. Places were introduced by Dedekind and Weber in the nineteenth century 21] with the purpose of constructing the Riemann surface associated with an affine curve from the field of functions of the curve. Also in that century, to study diophantine equations by using the Hensel's Lemma and solutions of the equations in the completions $\mathbb{Q}_{p}$, Hensel [31] considered $p$-adic valuations on the field of rational numbers, $\mathbb{Q}$, defined as $\nu_{p}(q):=$ $\alpha$, whenever $\mathbb{Q} \backslash\{0\} \ni q=p^{\alpha}(r / s)$ and $\operatorname{gcd}(r, p)=\operatorname{gcd}(s, p)=1$. The properties of $\nu_{p}$ give rise to the definition of valuation and its definition has to do with that of valuations centered at the completion of the local ring of a branch of a plane curve. In 1964, Hironaka [34 proved resolution of singularities in characteristic zero (some more recent references are $[49,22]$ ) and valuations were forgotten for a large period. However, activity in valuation theory has been increased in the last 
two decades, probably due to the lack of success in proving resolution in positive characteristic. Next we define the concept of valuation and some related objects.

Definition 2.1. A valuation of a commutative field $K$ is a surjective map $\nu: K^{*}(:=K \backslash\{0\}) \rightarrow G$, where $G$ is a totally ordered commutative group, such that for $f, g \in K^{*}$

- $\nu(f g)=\nu(f)+\nu(g)$.

- $\nu(f+g) \geq \min \{\nu(f), \nu(g)\}$ and the equality holds whenever $\nu(f) \neq \nu(g)$.

$G$ is usually named the value group of $\nu$ and the set $R_{\nu}:=\left\{f \in K^{*} \mid \nu(f) \geq\right.$ $0\} \cup\{0\}$ is a local ring, called the valuation ring of $\nu$, whose maximal ideal is $m_{\nu}:=\left\{f \in K^{*} \mid \nu(f)>0\right\} \cup\{0\}$. The rank of $\nu(\operatorname{rk}(\nu))$ is the Krull dimension of the ring $R_{\nu}$ and the dimension of the $\mathbb{Q}$-vector space $G \otimes_{\mathbb{Z}} \mathbb{Q}$ is the rational rank of $\nu(\operatorname{rrk}(\nu))$.

2.2. Value group and value semigroup. Along this paper we shall consider a Noetherian local domain $(R, m)$ whose field of fractions is $K$ and we shall assume that each valuation $\nu$ dominates $R$, that is $R \subset R_{\nu}$ and $R \cap m_{\nu}=m$. In this case, in addition to the two previous numerical invariants associated with $\nu$, we can consider the so-called transcendence degree of $\nu(\operatorname{tr} \cdot \operatorname{deg}(\nu))$, which is the transcendence degree of the field $k_{\nu}$ over $k$, where $k_{\nu}:=R_{\nu} / m_{\nu}$ and $k:=R / m$. Unless otherwise stated, we shall assume that $k$ is algebraically closed. The mentioned invariants are useful to classify valuations when $\operatorname{dim} R=2$. The value groups $G$ of valuations $\nu$ as above have been studied and classified [41, 42, $[\mathbf{5 2}, \mathbf{3 8}$. $G$ can be embedded in $\mathbb{R}^{n}$ with lexicographical ordering, $n$ being the dimension of $R$ and $\mathbb{R}$ the real numbers.

An interesting object which is not well-understood in general is the value semigroup of a valuation $\nu$ associated with $R$. This one is defined as

$$
S:=\{\nu(f) \mid f \in R \backslash\{0\}\} .
$$

Interesting data concerning ideal theory, singularities and topology are encoded by this semigroup. The two main facts which are known about it are:

1) The Abhyankar inequalities:

$$
\operatorname{rk}(\nu)+\operatorname{tr} \cdot \operatorname{deg}(\nu) \leq \operatorname{rrk}(\nu)+\operatorname{tr} \cdot \operatorname{deg}(\nu) \leq \operatorname{dim}(R) .
$$

Moreover, if $\operatorname{rk}(\nu)+\operatorname{tr} \cdot \operatorname{deg}(\nu)=\operatorname{dim} R$, then $G$ is isomorphic to $\mathbb{Z}^{\mathrm{rk}(\nu)}$ with lexicographical ordering and whenever $\operatorname{r.rk}(\nu)+\operatorname{tr} \cdot \operatorname{deg}(\nu)=\operatorname{dim} R$, then $G$ is isomorphic to $\mathbb{Z}^{\text {r.rk }(\nu)}$.

2) $S$ is a well-ordered subset of the positive part of the value group $G$ of ordinal type at most $\omega^{r k(\nu)}, \omega$ being the ordinal type of the set $\mathbb{N}$ of non-negative integers.

When $R$ is regular and $\operatorname{dim} R=1$, the semigroups $S$ are isomorphic to the natural numbers. The case $\operatorname{dim} R=2$ is also known; later we shall give more information about it. For higher dimension, very little is known. The second inequality in condition 1) gives a constraint on the value semigroup and recently, Cutkosky [14 has proved that the mentioned inequality and condition 2) do not characterize value semigroups on equicharacteristic Noetherian local domains. To do it he proves the forthcoming Proposition 2.2, which gives a new necessary condition for a semigroup to be a value semigroup. This allows him to provide an example of a well ordered sub-semigroup of the positive rational numbers $\mathbb{Q}_{+}$of ordinal type $\omega$ which is not a value semigroup of some equicharacteristic local domain. 
Proposition 2.2. With the above notations, let assume that $R$ is an equicharacteristic local domain and $\nu$ a valuation of $K$ that dominates $R$. Set $s_{0}:=$ $\min \{\nu(f) \mid f \in m \backslash\{0\}\}, n:=\operatorname{dim}_{k} m / m^{2}$ and $S_{\Psi}:=\nu(m \backslash\{0\}) \cap \Psi, \Psi$ being the convex subgroup of real rank 1 of $G$. Then,

$$
\operatorname{card}\left(S_{\Psi} \cap\left[0,(d+1) s_{0}\right)\right)<\left(\begin{array}{c}
n+d \\
n
\end{array}\right),
$$

for all nonnegative integer $d$, where we have set $[a, b):=\{c \in \Psi \mid a \leq c<b\}$, $a, b \in \Psi$.

Ideals in $R$ which are contraction of ideals in the valuation ring $R_{\nu}$ are named valuation ideals or $\nu$-ideals. The following result collects basic results on value semigroups and $\nu$-ideals. Recall that an order $\leq$ in a semigroup is called cancellative if $\alpha+\beta=\alpha+\gamma$ implies $\beta=\gamma$ and it is admissible if $\alpha+\gamma \leq \beta+\gamma$ whenever $\gamma \geq 0$ and $\alpha \leq \beta$.

Proposition 2.3. The value semigroup $S$ of a valuation $\nu$ of a field $K$, centered at $R$, is a cancellative, commutative, free of torsion, well-ordered semigroup with zero, where the associated order is admissible. Moreover, $F=\left\{P_{\alpha}\right\}_{\alpha \in S}$, where

$$
P_{\alpha}:=\{f \in R \backslash\{0\} \mid \nu(f) \geq \alpha\} \cup\{0\}
$$

is the family of $\nu$-ideals (in $R$ ) of the valuation $\nu$.

Proof. We shall prove that $S$ is free of torsion, $F$ is the family of $\nu$-ideals and, finally, that $S$ is well-ordered. The remaining properties are clear. Assume that $\nu(u) \neq 0, u \in K \backslash\{0\}$, then either $\nu(u)>0$ or $\nu\left(u^{-1}\right)>0$, so either $u \in m_{\nu}$ or $u^{-1} \in m_{\nu}$ and therefore either $u^{p} \in m_{\nu}$ or $u^{-p} \in m_{\nu}, p$ being a positive integer. Thus $\nu\left(u^{p}\right) \neq 0$ and the group spanned by $S, G(S)$ (which is $G$ ) is free of torsion. This proves that $S$ is also. $R$ is a Noetherian $\operatorname{ring}$ and then $\operatorname{rk}(\nu)<\infty$, so each $\nu$-ideal $\mathcal{I}$ is finitely generated. Consider a finite set of generators for $\mathcal{I}$ and set $\alpha$ the minimum of the values (by $\nu$ ) of these generators, then it is straightforward that $\mathcal{I}=P_{\alpha}$ and so $\mathcal{I} \in F$. Finally, $S$ is well-ordered because the family of $\nu$-ideals $F$ is also [52, App. 3].

Let $S$ be the value semigroup of a valuation $\nu$. $S$ satisfies that $(-S) \cap S=\{0\}$. This means that $\sum_{i=1}^{m} \alpha_{i}=0, \alpha_{i} \in S$, implies $\alpha_{i}=0$ for every index $i$. The length function of a semigroup $S, l: S \rightarrow \mathbb{N} \cup\{\infty\}$, is defined as $l(0)=0$ and, for $\alpha \neq 0$,

$$
l(\alpha):=\sup \left\{m \in \mathbb{N} \mid \alpha=\sum_{i=1}^{m} \alpha_{i}, \text { where } \alpha_{i} \in S \backslash\{0\}\right\} .
$$

In our case $l(\alpha)<\infty$ and therefore $S$ is generated by its irreducible elements, that is those elements in $S$ whose length is one. This is a consequence of the following result which can be deduced from the mentioned fact that $G$ can be embedded in $\mathbb{R}^{n}$ with the lexicographical ordering, $n$ being the dimension of $R$.

Proposition 2.4. 11] Let $\nu$ be a valuation and $S$ its value semigroup. Then, for each $\alpha \in S$, it happens that $t(\alpha)<\infty$, where

$$
t(\alpha):=\operatorname{card}\left\{\left\{\alpha_{i}\right\}_{i=1}^{m} \text { finite subset of } S \backslash\{0\} \mid \alpha=\sum_{i=1}^{m} \alpha_{i}\right\} .
$$

Generally speaking, the semigroups $S$ such that $t(\alpha)<\infty$ for all $\alpha \in S$ are called combinatorially finite. 


\section{Graded algebra of a valuation}

3.1. Graded algebra and generators. Let $\nu$ be a valuation of the field $K$ centered at the ring $R$. For each element $\alpha$ in the value semigroup $S$, consider the $\nu$-ideals $P_{\alpha}$ and $P_{\alpha}^{+}=\{f \in R \mid \nu(f)>\alpha\} \cup\{0\}$. The graded algebra of $R$ relative to $\nu$ is defined to be as the graded $k$-algebra

$$
\operatorname{gr}_{\nu} R:=\bigoplus_{\alpha \in S} \frac{P_{\alpha}}{P_{\alpha}^{+}}
$$

where the product of homogeneous elements is defined as follows: for $f \in P_{\alpha}$ and $g \in P_{\beta}, f$ modulo $P_{\alpha}^{+}$times $g$ modulo $P_{\beta}^{+}$is the class $f g$ modulo $P_{\alpha+\beta}^{+}$.

The field $k_{\nu}$ is an extension of the residue field of $\mathrm{R}, k$. There is a canonical field embedding of $k$ into $k_{\nu}$ and when this embedding is an isomorphism, one gets $\operatorname{dim}_{k} P_{\alpha} / P_{\alpha}^{+}=1$ for each $\alpha \in S$. In this case, if one fixes a nonzero element $\left[f_{\beta}\right] \in$ $P_{\beta} / P_{\beta}^{+}$for each $\beta \in \Lambda, \Lambda$ being the set of irreducible elements in $S$, and consider the $S$-graded $k$-algebra, $k_{\Lambda}[S]:=K\left[\left\{X_{\beta}\right\}_{\beta \in \Lambda}\right]$, where the $X_{\beta}$ are indeterminates of degree $\beta$, then there exists an epimorphism of graded $k$-algebras $\psi: k_{\Lambda}[S] \rightarrow \operatorname{gr}_{\nu} R$, given by $\Psi\left(X_{\beta}\right)=\left[f_{\beta}\right]$, which is homogeneous of degree zero and allows us to regard $\operatorname{gr}_{\nu} R$ as $k_{\Lambda}[S]$-module, ker $\psi$ being an ideal of $k_{\Lambda}[S]$ spanned by binomials.

Generally speaking $k$ is not isomorphic to $k_{\nu}$. In any case, the following property happens.

Proposition 3.1. For every $\alpha \in S, P_{\alpha} / P_{\alpha}^{+}$is a finite dimensional k-vector space.

Proof. The inclusion $m P_{\alpha} \subset P_{\alpha}^{+}$holds because $s_{0}(:=\min \{\nu(f) \mid f \in m\})>0$ and therefore $P_{\alpha} / P_{\alpha}^{+}$is a $k$-homomorphic image of $P_{\alpha} / m P_{\alpha}$ which is a $k$-vector space of finite dimension because $R$ is a Noetherian ring.

This result allows us to get by a recursive procedure a minimal system of generators of $\operatorname{gr}_{\nu} R, M=\left\{\left[f_{\gamma}\right]\right\}_{\gamma \in \Gamma}$, and attach to it an $S$-graded polynomial algebra $A[\nu]:=k\left[\left\{X_{\gamma}\right\}_{\gamma \in \Gamma}\right]$ that substitutes the former $k_{\Lambda}[S]$ for the general case. The procedure to obtain $M$ works by recurrence on the length of the elements in $S$ and it is based on the computation of certain bases of the vector spaces $P_{\alpha} / P_{\alpha}^{+}$with $l(\alpha)=n$ from the knowledge of the vector spaces $P_{\alpha} / P_{\alpha}^{+}$such that $l(\alpha)<n$. When $n=1$, we pick an arbitrary basis of $P_{\alpha} / P_{\alpha}^{+}$. Otherwise set

$$
\Omega_{\alpha}:=\left\{\left\{\alpha_{1}, \alpha_{2}, \ldots, \alpha_{r}\right\} \subseteq S \backslash\{0\}, r \geq 2 \mid \alpha=\sum_{i=1}^{r} \alpha_{i}\right\}
$$

and since, by recurrence, we know a basis of each vector space $P_{\alpha_{i}} / P_{\alpha_{i}}^{+}$, we are able to compute the following vector subspace of $P_{\alpha} / P_{\alpha}^{+}$:

$$
W_{\alpha}=\sum_{\left\{\alpha_{1}, \alpha_{2}, \ldots, \alpha_{r}\right\} \in \Omega_{\alpha}} \frac{P_{\alpha_{1}}}{P_{\alpha_{1}}^{+}} \cdot \frac{P_{\alpha_{2}}}{P_{\alpha_{2}}^{+}} \cdots \frac{P_{\alpha_{r}}}{P_{\alpha_{r}}^{+}}
$$

where the products of elements are in the algebra $\operatorname{gr}_{\nu} R$. Now take an arbitrary linearly independent set of $P_{\alpha} / P_{\alpha}^{+}$whose classes are a basis of the vector space $\frac{P_{\alpha}}{P_{\alpha}^{+}} / W_{\alpha}$. This set extends any basis of $W_{\alpha}$ to a basis of $P_{\alpha} / P_{\alpha}^{+}$. The set $M$ is obtained joining the bases of the vector spaces $P_{\alpha} / P_{\alpha}^{+}$such that $l(\alpha)=1$ with the described sets extending bases of the spaces $W_{\alpha}$ such that $l(\alpha)>1$. As a consequence, it holds that the elements $\gamma \in \Gamma$ are of the form $\gamma=\left(\beta, i_{\beta}\right)$ with 
$\beta \in S$ and $1 \leq i_{\beta} \leq \operatorname{dim}\left(\frac{P_{\beta}}{P_{\beta}^{+}} / W_{\beta}\right)$, where we have set $W_{\beta}=0$ when $l(\beta)=1$, and $A[\nu]$ is $S$-graded by setting $\operatorname{deg}\left(X_{\gamma}\right)=\operatorname{deg}(\gamma)=\beta \in S$, [1].

3.2. Minimal free resolution of $\operatorname{gr}_{\nu} R$. Denote by $A[\nu]_{\alpha}$ the homogeneous component of degree $\alpha$ of the ring $A[\nu]$ and consider the map

$$
\phi_{0}: A[\nu]=\bigoplus_{\alpha \in S} A[\nu]_{\alpha} \longrightarrow \operatorname{gr}_{\nu} R
$$

which maps $X_{\gamma}$ to $\left[f_{\gamma}\right]$; it is a homogeneous $k$-algebra epimorphism. Also consider the graded ideals $m[\nu]:=\bigoplus_{0 \neq \alpha \in S} A[\nu]_{\alpha}$ and $\mathcal{I}_{0}:=\operatorname{ker}\left(\phi_{0}\right)$, and a minimal homogeneous generating set of $\mathcal{I}_{0}, B=\cup_{\alpha \in S} B_{\alpha}, B_{\alpha}$ being the set of elements in $B$ of degree $\alpha$. By Nakayama's graded Lemma, the set of classes $\left[B_{\alpha}\right]$ of $B_{\alpha}$ in $\mathcal{I}_{0} / m[\nu] \mathcal{I}_{0}$ is a basis of the homogeneous component of degree $\alpha$ of $\mathcal{I}_{0} / m[\nu] \mathcal{I}_{0}$ and thus $\left[B_{\alpha}\right]$ and therefore $B_{\alpha}$ is finite since $A[\nu]_{\alpha}$ is a finite-dimensional vector space because $S$ is a combinatorially finite semigroup. This allows us to provide a degree 0 homogeneous homomorphism $\phi_{1}: L_{1}:=\oplus_{\alpha \in S}(A[\nu])^{l(\alpha)} \rightarrow A[\nu], l(\alpha)$ being the cardinality of $B_{\alpha}$ and recursively a minimal free resolution of $\operatorname{gr}_{\nu} R$ as $S$-graded $A[\nu]$-module:

$$
\text { (A.) }: \cdots \rightarrow L_{i} \stackrel{\phi_{i}}{\rightarrow} L_{i-1} \rightarrow \cdots \rightarrow L_{1} \stackrel{\phi_{1}}{\rightarrow} A[\nu] \rightarrow \operatorname{gr}_{\nu} R \rightarrow 0 .
$$

Write $N_{i}:=\operatorname{ker}\left(\phi_{i}\right)$, then the following result holds:

Proposition 3.2. [11]

(1) For every $i \geq 0$, there exists a homogeneous of degree 0 isomorphism of graded $A[\nu]$-modules between the ith Tor module $\operatorname{Tor}_{i}^{A[\nu]}\left(\operatorname{gr}_{\nu} R, k\right)$ and $L_{i} \bigotimes_{A[\nu]} k$.

(2) For each $\alpha \in S$, let denote the homogeneous component of degree $\alpha$ with the subindex $\alpha$, then

$$
\operatorname{dim}_{k}\left(\operatorname{Tor}_{i+1}^{A[\nu]}\left(\operatorname{gr}_{\nu} R, k\right)\right)_{\alpha}=\operatorname{dim}_{k} \frac{\left(N_{i}\right)_{\alpha}}{\left(m[\nu] N_{i}\right)_{\alpha}} .
$$

(3) There exists an isomorphism of $S$-graded modules between $\operatorname{Tor}_{i}^{A[\nu]}\left(k, \operatorname{gr}_{\nu} R\right)$ and the ith homology $H_{i}(G[\nu])$ of an augmented Koszul complex of $\operatorname{gr}_{\nu} R$ modules.

As a consequence of the commutative property of the Tor functor and from item (2), the number of homogeneous elements of degree $\alpha$ in a minimal set of homogeneous generators of the ith syzygy module of $\operatorname{gr}_{\nu} R$ as $A[\nu]$-module is $\operatorname{dim}_{k}\left(H_{i}(G[\nu])_{\alpha}\right.$.

The graded algebra relative to a valuation seems to be a useful tool to study the local uniformization problem. This consists of, given the local ring of an algebraic variety (assuming that it is an integral domain), finding, for each valuation $\nu$ centered at $R$, a regular local $R$-algebra $R^{\prime}$ essentially of finite type over $R$ and contained in $R_{\nu}$. In [47, Teissier proposes that $R^{\prime}$ might be obtained from an affine chart of a proper algebraic map $Z \rightarrow \operatorname{Spec} R$ which would be described as a proper and birational toric map with respect to some system of generators of the maximal ideal of $R$. An idea to do this would be to view $R$ as a deformation of the graded ring $\operatorname{gr}_{\nu} R$ with respect to the filtration associated with the valuation and to obtain the uniformization of the valuation $\nu$ as a deformation of the valuation induced by 
$\nu$ on $\operatorname{gr}_{\nu} R$; the motivating example is the case of complex plane branches which has been studied by Goldin and Teissier as deformations of monomial curves.

Without doubt, the most interesting valuations from a geometric point of view are the so-called divisorial valuations because they are attached to irreducible exceptional divisors of some birational map. Next we state the definition.

Definition 3.3. Let us assume that $\operatorname{dim} R=n$. A valuation $\nu$ of $K$ centered at $R$ is called to be divisorial whenever its rank is 1 and its transcendence degree is $n-1$.

When $n=2$, the graded algebra $\operatorname{gr}_{\nu} R$ of a divisorial valuation is Noetherian. Notwithstanding, this does not happen in higher dimension. For instance, let $R$ be a 3-dimensional local regular ring and blow-up $X_{0}=\operatorname{Spec} R$ at its maximal ideal $m_{0}$. Let $X_{1}$ be the obtained variety. Consider the cubic with equation $x^{2} z+x y^{2}+y^{3}=0$ on the obtained exceptional divisor $E_{1}:=\operatorname{Proj}(k[x, y, z])$ and a sequence of $n \geq 10$ point blowing-ups $X_{n} \rightarrow \cdots \rightarrow X_{0}$ centered at $m_{0}$ and at points $m_{i}$ in $X_{i}, 1 \leq i \leq n$, on the last obtained exceptional divisor $E_{i}$ and on the strict transform of the cubic. Denote by $\nu$ the divisorial valuation given by the divisor $E_{n}$ and set $R_{i}:=\mathcal{O}_{X_{i}, m_{i}}$. It is not difficult to prove that $R_{1}=k\left[a_{1}, b_{1}, c_{1}\right]_{\left(a_{1}, b_{1}, c_{1}\right)}$, where $a_{1}=x, b_{1}=y / x$ and $c_{1}=(x / z)+(y / x)^{2}+(y / x)^{3}$. If $A_{1}, B_{1}, C_{1}$ are, respectively, the initial forms of $a_{1}, b_{1}, c_{1}$ on $\operatorname{gr}_{\nu} R_{1}=k\left[A_{1}, B_{1}, C_{1}\right]$, then we can state

Proposition 3.4. 13. The family $A_{1}, A_{1} B_{1}, A_{1}^{3} C_{1}, A_{1} B_{1}^{2}, A_{1}^{2} B_{1}^{5}, A_{1}^{3} B_{1}^{8}, \ldots$, $A_{1}^{i} B_{1}^{3 i-1}, \ldots$ is a minimal system of generators of $g r_{\nu} R \subset g r_{\nu} R_{1}$. As a consequence $\operatorname{gr}_{\nu} R$ is not Noetherian.

An interesting number associated with a divisorial valuation $\nu$ is the volume. In this case $\mathbb{Z}$ is the value group of $\nu$ and by definition, the volume of $\nu$ is

$$
\operatorname{vol}(\nu):=\limsup _{\alpha \in \mathbb{N}} \frac{\operatorname{length}\left(R / P_{\alpha}\right)}{\alpha^{n} / n !} .
$$

This definition corresponds to the analogue of the Samuel multiplicity for an $m$ primary ideal $p \subseteq R$ :

$$
e(p):=\limsup _{\alpha \in \mathbb{N}} \frac{\operatorname{length}\left(R / p^{\alpha}\right)}{\alpha^{n} / n !} .
$$

It is known that the multiplicity is always an integer number and also $[\mathbf{2 3}$ that

$$
\operatorname{vol}(\nu)=\lim _{\alpha \rightarrow \infty}\left(e\left(P_{\alpha}\right) / \alpha^{n}\right) .
$$

However the volume of a divisorial valuation is not always an integer number although it is rational when its graded algebra is Noetherian. As a consequence valuations with irrational volume provide non-finitely generated attached graded algebras. For an example, see [37.

\section{Plane valuations}

4.1. Definition and geometric sense. From this section on we shall consider plane valuations, notwithstanding from time to time we shall speak about other types of valuations. We start this section with the definition.

Definition 4.1. A plane valuation is a valuation of a field $K$ which is the fraction field of a two-dimensional Noetherian local regular ring $R$ and is centered at $R$. 
Zariski in 51 classified plane valuations by attending invariants as the rank and the rational rank. By using previous results by Zariski, Spivakovsky [46] gives the following geometric view of plane valuations.

THEOREM 4.2. There is a one to one correspondence between the set of plane valuations (of $K$ centered at $R$ ) and the set of simple sequences of point blowing-ups of the scheme Spec $R$.

The correspondence in Theorem 4.2 works as follows: each valuation $\nu$ is associated with the sequence

$$
\pi: \cdots \longrightarrow X_{N+1} \stackrel{\pi_{N+1}}{\longrightarrow} X_{N} \longrightarrow \cdots \longrightarrow X_{1} \stackrel{\pi_{1}}{\longrightarrow} X_{0}=X=\operatorname{Spec} R,
$$

where $\pi_{i+1}$ is the blowing-up of $X_{i}$ at the unique closed point $p_{i}$ of the exceptional divisor obtained after the blowing-up $\pi_{i}, E_{i}$, which satisfies that $\nu$ is centered at the local ring $\mathcal{O}_{X_{i}, p_{i}}\left(:=R_{i}\right)$.

Theorem 4.2 allows Spivakovsky to give a classification of plane valuations which improves the Zariski's one and it is based in the form of the so-called dual graph of the sequence $\pi$. This graph is a (in general, infinite) tree whose vertices represent the strict transforms in $X_{l}, l$ large enough, of the divisors $E_{i}$ (also named $\left.E_{i}\right)$ and two vertices are joined by an edge whenever these strict transforms intersect. Set $\mathcal{C}_{\nu}=\left\{p_{i}\right\}_{i \geq 0}$ the configuration of infinitely near points determined by $\nu$. We say that $p_{i}$ is proximate to $p_{j}$ (denoted by $p_{i} \rightarrow p_{j}$ ) whenever $i>j$ and $p_{i}$ belongs either to $E_{j+1}$ or to the strict transform of $E_{j+1}$ at $X_{i}$ and $p_{i}$ is said to be satellite if there exists $j<i-1$ such that $p_{i} \rightarrow p_{j}$. Valuations whose associated sequence (4.1) is finite are exactly the divisorial ones. The dual graph shape of a divisorial (plane) valuation is that of Figure 1.

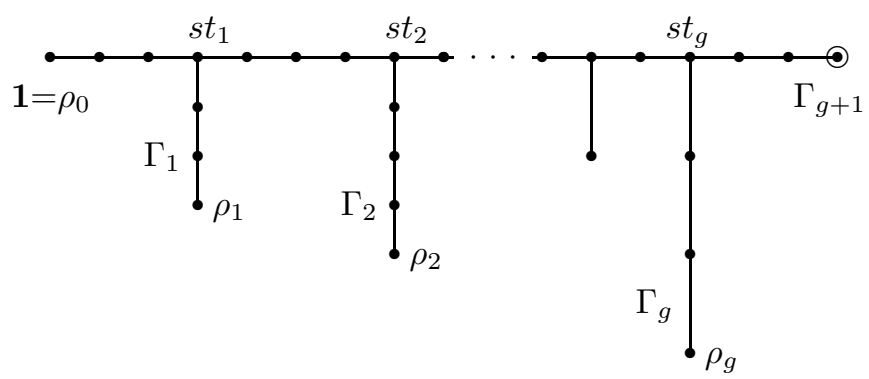

FiguRE 1. The dual graph of a divisorial valuation

The dual graph is not suitable when we desire to get parametric equations for computing valuations. Furthermore, the classical theory for curves uses, for this purpose, Puiseux exponents that only work for zero characteristic. Next, we recall the Spivakovsky's classification in terms of the so-called Hamburger-Noether expansions of valuations. These expansions provide parametric equations for plane valuations [27 and have been used in [18 to study saturation with respect to this type of valuations.

4.2. Hamburger-Noether expansions and classification of plane valuations. Let $\nu$ be a plane valuation and take $\{u, v\}$ a regular system of parameters for the ring $R$. Assume that $\nu(u) \leq \nu(v)$. This means that there exists an element $a_{01} \in k$ such that the set $\left\{u_{1}=u, v_{1}=(v / u)-a_{01}\right\}$ constitutes a 
regular system of parameters for the ring $R_{1}$. If, now, $\nu(u) \leq \nu\left(v_{1}\right)$ holds, then we repeat the above operation and we keep doing the same thing until we get $v=a_{01} u+a_{02} u^{2}+\cdots+a_{0 h} u^{h}+u^{h} v_{h}$, where either $\nu(u)>\nu\left(v_{h}\right)$ or $\nu\left(v_{h}\right)=0$, or $v=a_{01} u+a_{02} u^{2}+\cdots+a_{0 h} u^{h}+\cdots$, with infinitely many steps. In the last two cases, we have got the Hamburger-Noether expansion for $\nu$, obtaining $R_{\nu}=R_{h}$ when $\nu\left(v_{h}\right)=0$. Otherwise, set $w_{1}:=v_{h}$ and reproduce the above procedure for the regular system of parameters $\left\{w_{1}, u\right\}$ of $R_{h}$. The procedure can continue indefinitely or we can obtain a last equality. In any case, we attach to $\nu$ a set of expressions called the Hamburger-Noether expansion of the valuation $\nu$ in the regular system of parameters $\{u, v\}$ of the ring $R$ which provides a regular system of parameters for each local ring $R_{i}$ given by the sequence $\pi$ described in (4.1) and it has the form given in Figure 2

$$
\begin{aligned}
v= & a_{01} u+a_{02} u^{2}+\cdots+a_{0 h_{0}} u^{h_{0}}+u^{h_{0}} w_{1} \\
u= & w_{1}^{h_{1}} w_{2} \\
\vdots & \vdots \\
w_{s_{1}-2}= & w_{s_{1}-1}^{h_{s_{1}-1}} w_{s_{1}} \\
w_{s_{1}-1}= & a_{s_{1} k_{1}} w_{s_{1}}^{k_{1}}+\cdots+a_{s_{1} h_{s_{1}}} w_{s_{1}}^{h_{s_{1}}}+w_{s_{1}}^{h_{s_{1}}} w_{s_{1}+1} \\
\vdots & \vdots \\
w_{s_{g}-1}= & a_{s_{g} k_{g}} w_{s_{g}}^{k_{g}}+\cdots+a_{s_{g} h_{s_{g}}} w_{s_{g}}^{h_{s_{g}}}+w_{s_{g}}^{h_{s_{g}}} w_{s_{g}+1} \\
\vdots & \vdots \\
w_{i-1}= & w_{i}^{h_{i}} w_{i+1} \\
\vdots & \vdots \\
\left(w_{z-1}=\right. & \left.w_{z}^{\infty}\right) .
\end{aligned}
$$

Figure 2. Hamburger-Noether expansion of a plane valuation

The nonnegative integers $\left\{s_{j}\right\}_{j=0}^{g}$ correspond to rows with some nonzero $a_{s_{j} l}$ (called free ones and that are those associated with the non-satellite blowing-up points), $g \in \mathbb{N} \cup\{\infty\}$ and $k_{j}=\min \left\{n \in \mathbb{N} \mid a_{s_{j}, n} \neq 0\right\}$. Thus, plane valuations can be classified in the following five types which we name with a letter or as in 24 .

- Type A or divisorial valuations. Their Hamburger-Noether expansion is finite and their last row has the following shape

$$
w_{s_{g}-1}=a_{s_{g} k_{g}} w_{s_{g}}^{k_{g}}+\cdots+a_{s_{g} h_{s_{g}}} w_{s_{g}}^{h_{s_{g}}}+w_{s_{g}}^{h_{s_{g}}} w_{s_{g}+1},
$$

where $g<\infty, h_{s_{g}}<\infty, w_{s_{g}+1} \in R_{\nu}$ and $\nu\left(w_{s_{g}+1}\right)=0$.

- Type $B$ or curve valuations. Their Hamburger-Noether expansion has a last equality associated with an infinite sum like this $w_{s_{g}-1}=\sum_{i=k_{g}}^{\infty} a_{s_{g}} w_{s_{g}}^{i}$. Here $g<\infty$ and there exists a positive integer $i_{0}$ such that $p_{i}$ is free for all $i>i_{0}$.

- Type $C$ or exceptional curve valuations. Their Hamburger-Noether expansion has a last free row like (4.2) and, after, finitely many non-free rows with the shape

$$
\begin{array}{ccc}
w_{s_{g}} & = & w_{s_{g}+1}^{h_{s_{g}+1}} w_{s_{g}+2} \\
\vdots & & \vdots \\
w_{z-1} & = & w_{z}^{\infty} .
\end{array}
$$




\begin{tabular}{||c|c|c|c|c||}
\hline type & subtype & rk & r.rk & tr.deg \\
\hline \hline A & - & 1 & 1 & 1 \\
\hline B & I & 1 & 1 & 0 \\
& II & 2 & 2 & 0 \\
\hline C & - & 2 & 2 & 0 \\
\hline D & - & 1 & 2 & 0 \\
\hline E & - & 1 & 1 & 0 \\
\hline
\end{tabular}

TABLE 1

In this case, $g<\infty, s_{g}<\infty$ and there exists a positive integer $i_{0}$ such that $p_{i} \rightarrow p_{i_{0}}$ for all $i>i_{0}$.

- Type D or irrational valuations. A plane valuation will be called of type D, whenever its Hamburger-Noether expansion has a last free row like (4.2) followed by infinitely many rows with the shape $w_{i-1}=w_{i}^{h_{i}} w_{i+1} \quad\left(i>s_{g}\right)$. Now $g<\infty$ and there exists a positive integer $i_{0}$ such that $p_{i}$ is a satellite point for all $i \geq i_{0}$ but $\nu$ is not a type $\mathrm{C}$ valuation.

- Type E or infinitely singular valuations. When the Hamburger-Noether expansion of a plane valuation repeats indefinitely the basic structure, then the valuation is called to be of type $E$. This means that the sequence $\mathcal{C}_{\nu}$ alternates indefinitely blocks of 1 free and $(1 \leq) l(<\infty)$ non-free rows. Here $g=z=\infty$.

This classification does not depend on the regular system of parameters we choose on $R$. Table 1 relates our classification with the invariants of $\nu$ above defined. Notice that classical invariants provide a refinement of type B valuations. We also add that in 24 the real-valued class of plane valuations is interpreted in a rooted metric tree in such a way that the valuations are partially ordered and there is a unique path from any valuation to any other, being this path isometric to a real interval.

4.3. Other invariants of plane valuations. Let $\nu$ be a plane valuation and $\left\{m_{i}\right\}_{i \geq 0}$ the family of maximal ideals of the rings $R_{i}$ of the sequence (4.1). We attach to $\nu$ the following data:

- The sequence $\left\{\min \left\{\nu(f) \mid f \in m_{i} \backslash\{0\}\right\}_{i \geq 0}\right.$, that we call sequence of values of $\nu$.

- The sequence $\left\{\beta_{j}^{\prime}\right\}_{0 \leq j<g+2}$ that we name Puiseux exponents of $\nu$ and, with the convention $1 / \infty=0$, it is defined as $\beta_{0}^{\prime}:=1$ and

$$
\beta_{j+1}^{\prime}:=h_{s_{j}}-k_{j}+1+\frac{1}{h_{s_{j}+1}+\frac{1}{\ddots}} .
$$

- Set $\beta_{j}^{\prime}=p_{j} / n_{j}$ with $\operatorname{gcd}\left(p_{j}, n_{j}\right)=1$ and $r_{i}=\nu\left(w_{i}\right)$ and $e_{j}=\nu\left(w_{s_{j}}\right)$ for $0 \leq j<g+1$ and $i \geq 0$. Define

$$
\beta_{j+1}:=\beta_{j}+\left(h_{s_{j}}-k_{j}\right) e_{j}+r_{s_{j}+1}
$$

and

$$
\bar{\beta}_{j+1}:=n_{j} \bar{\beta}_{j}+\left(h_{s_{j}}-k_{j}\right) e_{j}+r_{s_{j}+1} .
$$

Then the sequence $\left\{\beta_{j}\right\}_{j=0}^{g+1}$ is called to be the characteristic sequence and the sequence $\left\{\bar{\beta}_{j}\right\}_{j=0}^{g+1}$ the sequence of maximal contact values both of $\nu$. 
Last three sequences are infinite in case $\mathrm{E}$, and in case B we only consider sub-indices from $j=0$ to $j=g$ although in case B-II we add to $\left\{\bar{\beta}_{j}\right\}_{j=0}^{g}$ the minimum element in the value semigroup $S$ with non-zero first coordinate, denoted by $\bar{\beta}_{g+1}$. The main result concerning maximal contact values is that they are a set of generators of $S$. Moreover, if we delete the last one $\bar{\beta}_{g+1}$ in type A valuations, we get a minimal set of generators for $S$. We can determine the type of a valuation if we know either its sequence of values or its characteristic exponents or its maximal contact values, but this does not happen with the Puiseux exponents or with the semigroup. When one knows the type of the valuation, the following result holds.

Proposition 4.3. 18] Assume that $\nu$ is a plane valuation and that we know which is its type. Then any of the following invariants can be computed from whichever of the others: sequence of values, Puiseux exponents, maximal contact values, characteristic exponents, and semigroup $S$ of the valuation (or pair $\left.\left(S, \bar{\beta}_{g+1}\right)\right)$.

\section{Poincaré series of the graded algebra}

5.1. General case. For a while, we consider a non-necessarily plane valuation $\nu$. The Poincaré series (of the graded algebra) of $\nu$ is the formal power series in the indeterminate $t$ :

$$
H_{\mathrm{gr}_{\nu} R}(t):=\sum_{\alpha \in S} \operatorname{dim}_{k} \frac{P_{\alpha}}{P_{\alpha}^{+}} t^{\alpha},
$$

which, according Proposition 3.1. belongs to the power series ring on $S$.

Let us assume that the value group of $\nu$ is isomorphic to the integer numbers. This is equivalent to say that the ring $R_{\nu}$ is Noetherian [52, however the algebra $\operatorname{gr}_{\nu} R$ may be non-Noetherian and its Poincaré series a non-rational function, even $\operatorname{gr}_{\nu} R$ might be non-Noetherian but $H_{\mathrm{gr}_{\nu} R}(t)$ a rational function. When the ring $R$ is 2-dimensional and normal and $\nu$ is a divisorial valuation, this series is the generating function of a sequence of integers which is residually equal to the sum of a polynomial with a periodic function (see [16] and [13]). An explicit computation for the plane divisorial case can be found in [25]; as we shall see, the Poincaré series is very close to that attached to the semigroup of the valuation or to the Poincare series of the analytically irreducible germ of curve provided by a general element of the valuation [30. One can found many papers studying Poincaré series for singularities (which need not to correspond to the irreducible case), some of them are $8,9,15,28,40,45$.

5.2. The plane case. An important concept for studying plane valuations is that of generating sequence. This concept was introduced in $4 \mathbf{4 6}$ and the existence of those sequences is discussed in [29. Notice that the hypothesis of 2dimensionality of $R$ is not necessary to define this concept.

Definition 5.1. A sequence $\left\{r_{j}\right\}_{j \in \mathcal{J}}$ of elements in the maximal ideal $m$ of $R$ is called to be a generating sequence (relative to $R$ ) of a valuation $\nu$ if, for any element $\alpha \in S, P_{\alpha}$ is spanned by the set

$$
\left\{\prod_{j \in \mathcal{J}_{0} \subseteq \mathcal{J}, \mathcal{J}_{0}}{ }_{\text {finite }} r_{j}^{a_{j}} \mid a_{j} \in \mathbb{N}, a_{j}>0 \text { and } \sum_{j \in \mathcal{J}_{0}} a_{j} \nu\left(r_{j}\right) \geq \alpha\right\} .
$$


Assume that the Hamburger-Noether expansion of $\nu$ is that given in Figure 2. Set $q_{0}=u, q_{1}=v$ and, for $1<j<g+2$, let $q_{j}$ be the defining equation of some analytically irreducible germ of curve on Spec $R$ whose Hamburger-Noether expansion in the basis $\left\{\bar{u}=u+\left(q_{j}\right), \bar{v}=v+\left(q_{j}\right)\right\}$ of $\hat{R} /\left(q_{j}\right)[\mathbf{5}, \hat{R}$ being the $m$-adic completion of $R$, is

$$
\begin{aligned}
\bar{v}= & a_{01} \bar{u}+a_{02} \bar{u}^{2}+\cdots+a_{0 h_{0}} \bar{u}^{h_{0}}+\bar{u}^{h_{0}} \bar{w}_{1} \\
\bar{u}= & \bar{w}_{1}^{h_{1}} \bar{w}_{2} \\
& \vdots \\
\vdots & \vdots \\
\bar{w}_{s_{j-1}-1}= & a_{s_{j-1} k_{j-1}} \bar{w}_{s_{j-1}}^{k_{j-1}}+\cdots+a_{s_{j-1} h_{s_{j-1}}} \bar{w}_{s_{j-1}}^{h_{s_{j-1}}}+\cdots .
\end{aligned}
$$

In [46] it is proved that any generating sequence of a divisorial valuation contains a subsequence $\left\{q_{j}\right\}_{j=0}^{g}$. Moreover, this set is a minimal generating sequence (no subset of it is a generating sequence) whenever the dual graph of $\nu$ (Figure 1) contains no subgraph $\Gamma_{g+1}$ (or equivalently $h_{s_{g}}-k_{g}=0$ ); otherwise, $\left\{q_{j}\right\}_{j=0}^{g+1}$ is a minimal generating sequence. Now, let $\nu$ be a valuation of type $C$ or $D$. In both cases a minimal generating sequence of $\nu$ is of the form $\left\{q_{j}\right\}_{j=0}^{g+1}$. In the first type of valuations $\nu\left(q_{j}\right)(0 \leq j<g+1)$ are data lying on the line that joins the origin to $\nu\left(q_{0}\right)$, but $\nu\left(q_{g+1}\right)$ does not satisfy this property. With respect to the second type, $\nu\left(q_{j}\right) \in \mathbb{Q}$ whenever $0 \leq j<g+1$, but $\nu\left(q_{g+1}\right) \in \mathbb{R} \backslash \mathbb{Q}$. Whenever $\nu$ is a type $E$ valuation, a minimal generating sequence of $\nu$ is an infinite sequence of the form $\left\{q_{j}\right\}_{0 \leq j}$. However neither all valuations have minimal generating sequences nor every element in a minimal generating sequence must be analytically irreducible. Valuations of type B-II which admit minimal generating sequences with this last condition are called of type B-II-a and the remaining ones will be of type B-II-b. Type B-I valuations do not admit minimal generating sequences. To understand this fact, we have to consider an element $q_{g+1}$ which, in general, will be in $\hat{R} . q_{g+1}$ will be the defining equation of $\nu$. If $q_{g+1}$, up to multiplication by an unit, belongs to $R$, then we are speaking about a valuation of type B-II-a and $\left\{q_{j}\right\}_{j=0}^{g+1}$ is a minimal generating sequence of $\nu$. When there exists an element in $R$ which factorizes in $\hat{R}$ as a product which contains $q_{g+1}$ as a non-trivial factor, $\nu$ is of type B-II-b and otherwise it is of type B-I [46, Section 9, case 4].

One important property for the generating sequences is given in the next

TheOREM 5.2. 25, 27 Let $\nu$ be a type A, B-II-a, C or D plane valuation. Then a set $\left\{r_{j}\right\}_{j \in \mathcal{J}}$ of elements in the maximal ideal $m$ of $R$ is a generating sequence of $\nu$ if, and only if, the $k$-algebra $g r_{\nu} R$ is spanned by the classes defined by the elements $r_{j}$ in $g r_{\nu} R$. In addition, when $\nu$ is of type $E$, it is also true that the classes defined by the elements $r_{j}$ in $g r_{\nu} R$ span that algebra whenever $\left\{r_{j}\right\}_{j \in \mathcal{J}}$ is a generating sequence.

5.2.1. The divisorial case. Assume that $\nu$ is a divisorial plane valuation and pick a generating sequence of $r+1$ elements. Recalling Section 3 , consider the ring $A[\nu]=k\left[X_{0}, \ldots, X_{r}\right]$ and the exact sequence of graded algebras $0 \rightarrow \mathcal{I}_{0} \rightarrow A[\nu] \stackrel{\phi_{0}}{\rightarrow}$ $\operatorname{gr}_{\nu} R \rightarrow 0$ which gives rise to the following equality of Poincaré series of graded algebras $H_{\mathrm{gr}_{\nu} R}(t)=H_{A[\nu]}(t)-H_{\mathcal{I}_{0}}(t)$. With the help of generating sequences and making use of the Hamburger-Noether expansion properties, one can get the following result proved in $[\mathbf{2 5}$. 
THEOREM 5.3. Let $\nu$ be a plane divisorial valuation, $\left\{\bar{\beta}_{j}\right\}_{j=0}^{g+1}$ its maximal contact values, $e_{j}=\operatorname{gcd}\left(\bar{\beta}_{0}, \bar{\beta}_{1}, \ldots, \bar{\beta}_{j}\right)$ and $n_{j}=e_{j-1} / e_{j}$. Then, $H_{\mathrm{gr}_{\nu} R}(t)=$ $H_{S}(t) H^{\prime}(t)$, where

$$
H_{S}(t):=\sum_{\alpha \in S} t^{\alpha}=\frac{1}{1-t^{\bar{\beta}_{0}}} \prod_{j=1}^{g} \frac{1-t^{n_{j} \bar{\beta}_{j}}}{1-t^{\bar{\beta}_{j}}}
$$

is the Poincaré series of the value semigroup of the valuation and $H^{\prime}(t)=\frac{1}{1-t^{\beta} g+1}$.

As a consequence the Poincaré series and the dual graph of a plane divisorial valuation are equivalent data.

The case when $k$ is infinite but it needs not to be algebraically closed has been recently treated in $[\mathbf{3 6}$ where it is also introduced a motivic Poincaré series.

5.2.2. The remaining plane cases. Assume now that $\nu$ is a non-divisorial plane valuation. Then, $\operatorname{dim} P_{\alpha} / P_{\alpha}^{+}=1$ for any $\alpha \in S$ and then $\operatorname{gr}_{\nu} R$ is a $k$-algebra isomorphic to the algebra of the semigroup $S$. Thus the Poincaré series for $S$ (that is, the series $H_{S}(t)$ defined in Theorem 5.3) and for $\operatorname{gr}_{\nu} R$ coincide. With notations as in Section 4, from [18, 1.10.5] it is not difficult to prove that

$$
H_{\mathrm{gr}_{\nu} R}(t)=\frac{1}{1-t^{\bar{\beta}_{0}}} \prod_{j=1}^{g} \frac{1-t^{n_{j} \bar{\beta}_{j}}}{1-t^{\bar{\beta}_{j}}} \frac{1}{1-t^{\bar{\beta}_{g+1}}},
$$

except in cases B-I and E. In these cases

$$
H_{\operatorname{gr}_{\nu} R}(t)=\frac{1}{1-t^{\bar{\beta}_{0}}} \prod_{j=1}^{g} \frac{1-t^{n_{j} \bar{\beta}_{j}}}{1-t^{\bar{\beta}_{j}}},
$$

and $g=\infty$ whenever $\nu$ is of type E.

\section{Graded algebra and Poincaré series of finite families valuations}

6.1. Families of valuations whose value group is $\mathbb{Z}$. Throughout this sub-section, we consider a family $V=\left\{\nu_{i}\right\}_{i=1}^{m}$ of valuations of the quotient field $K$ of a Noetherian local domain $(R, m)$ centered at $R$ such that $\mathbb{Z}$ is the value group of each $\nu_{i}, i \leq i \leq m$. It is known that these valuations are of rank 1 . For $\underline{\alpha}, \underline{\beta} \in \mathbb{N}^{m}$, we say $\underline{\alpha} \geq \underline{\beta}$ whenever $\underline{\alpha}-\beta \in \mathbb{N}^{m}$. Write $\underline{\nu}(f)=\left(\nu_{1}(f), \nu_{2}(f), \ldots, \nu_{m}(f)\right)$ for $f \in K$ and define the ideal in $\bar{R}$,

$$
P_{\underline{\alpha}}^{V}:=\{f \in R \mid \underline{\nu}(f) \geq \underline{\alpha}\} \cup\{0\} .
$$

Now we introduce the concepts of graded algebra and Poincaré series for our family $V$ of valuations. Set $\underline{e}_{i} \in \mathbb{N}^{m}$, the $m$-tuple such that all its coordinates are zero but the $i$ th one which is 1 , furthermore $\underline{e}_{\leq i}:=\sum_{j=0}^{i} \underline{e}_{j}, \underline{e}_{\leq 0}:=0 \in \mathbb{N}^{m}$ and $\underline{e}:=\underline{e}_{\leq m}$.

DEFINITION 6.1. We define the graded algebra associated with the family $V$ as the graded $k$-algebra

$$
\operatorname{gr}_{V} R=:=\bigoplus_{\underline{\alpha} \in \mathbb{N}^{m}} \frac{P_{\underline{\alpha}}^{V}}{P_{\underline{\alpha}}^{V}+\underline{e}} .
$$


Since $\frac{P_{\underline{\alpha}}^{V}}{P_{\underline{\alpha}}^{V}+\underline{e}} \cdot \frac{P_{\underline{\alpha}}^{V}}{P_{\underline{\beta}+\underline{e}}^{V}} \subseteq \frac{P_{\underline{\alpha}}^{V}+\underline{\beta}}{P_{\underline{\alpha}}^{V}+\underline{\beta}+\underline{e}}$ when $\underline{\alpha}, \underline{\beta} \in \mathbb{N}^{m}, \operatorname{gr}_{V} R$ is a well-defined $\mathbb{N}^{m}$ graded algebra. On the other hand, Nakayama's Lemma proves that, for each $\underline{\alpha} \in \mathbb{N}^{m}, \frac{P_{\alpha}^{V}}{P_{\underline{\alpha}+e}^{V}}$ is a finite dimensional $k$-vector space. Denote $\underline{t}=\left(t_{1}, t_{2}, \ldots, t_{m}\right)$ and $\underline{t} \underline{\underline{\alpha}}=t_{1}^{\underline{\alpha_{1}}} t_{2}^{\alpha_{2}} \cdots t_{m}^{\alpha_{m}}$.

Definition 6.2. The multi-graded (or multi-index) Poincaré series of the graded algebra $\operatorname{gr}_{V} R$ is defined to be

$$
H_{\operatorname{gr}_{V} R}\left(t_{1}, t_{2}, \ldots, t_{m}\right)=H_{\operatorname{gr}_{V} R}(\underline{t}):=\sum_{\underline{\alpha} \in \mathbb{N}^{m}} \operatorname{dim}_{k}\left(P_{\underline{\alpha}} / P_{\underline{\alpha}+e}\right) \underline{t} \underline{\underline{\alpha}} \in \mathbf{Z}\left[\left[t_{1}, \ldots, t_{m}\right]\right],
$$

where $\operatorname{dim}_{k}$ means dimension as $k$-vector space.

Definition 5.1 can be extended by stating that a family $\Lambda=\left\{r_{j}\right\}_{j \in \mathcal{J}}$ of elements in $m$ is a generating sequence (or a generating set) of $V$ whenever $P_{\underline{\alpha}}^{V}$ is spanned by the set given in (5.1) but replacing $\nu$ with $\underline{\nu}$ and $\alpha$ with $\underline{\alpha}$. This allows us to give the following definition for families of valuations $V=\left\{\nu_{i}\right\}_{i=1}^{m}$ as above.

Definition 6.3. A finite family of valuations $V$ is said to be monomial with respect to some system of generators $\Lambda=\left\{r_{j}\right\}_{j \in \mathcal{J}}$ of the maximal ideal $m$ of $R$ if $\Lambda$ is a generating set of $V$.

In these conditions, we have the following extended version of Theorem 5.2

Proposition 6.4. Let $V=\left\{\nu_{i}\right\}_{i=1}^{m}$ be a family of valuations of $K$ centered at $R$ whose value group is $\mathbb{Z}$. Assume that there exists a finite generating sequence for some valuation of $V$. Then, a system of generators $\Lambda=\left\{r_{j}\right\}_{j \in \mathcal{J}}$ of the maximal ideal $m$ is a generating set of the family $V$ if, and only if, the $k$-algebra $g r_{V} R$ is generated by the set $\left\{\left[r_{j}\right]\right\}_{j \in \mathcal{J}}$, where $\left[r_{j}\right]$ denotes the coset that $r_{j}$ defines in $\operatorname{gr}_{V} R$ and the meaning of the expression "coset defined by $r_{j}$ " is clarified in the remark after the proof.

Proof. 12 Along this proof, we set $P$ instead $P^{V}, \gamma$ will denote elements in $\mathbb{N}^{s}, s \geq 0$, whose $j$ th component is $\gamma_{j}, r^{\gamma}$ will stand for $\prod_{j=1}^{s} r_{j}^{\gamma_{j}}$ and $[r]^{\gamma}$ will be $\prod_{j=1}^{s}\left[r_{j}\right]^{\gamma_{j}}$. Assume that $\Lambda$ is a generating set for $V$. Let $f+P_{\underline{\alpha}+\underline{e}}$ be a nonzero element in $\operatorname{gr}_{V} R$, then $f \in P_{\underline{\alpha}}$ and so $f$ is in the ideal generated by the set given in (5.1) -with $\underline{\alpha}$ and $\underline{\nu}$ instead of $\alpha$ and $\nu$ - which we denote by $P_{\underline{\alpha}}^{\prime}$. Therefore,

$$
f=\sum_{\gamma \in Q_{0} \subseteq Q_{\underline{\alpha}}, Q_{0}} a_{\gamma} r^{\gamma},
$$

where $a_{\gamma} \in k$ and $Q_{\underline{\alpha}}=\left\{\gamma \mid s \in \mathbb{N}, \underline{\nu}\left(r^{\gamma}\right) \geq \underline{\alpha}\right\}$. As a consequence, $f+P_{\underline{\alpha}+\underline{e}}=$ $\sum_{\gamma \in Q_{0}^{\prime}} a_{\gamma}[r]^{\gamma}$, where $Q_{0}^{\prime}=\left\{\gamma \in Q_{0} \mid \underline{\nu}\left(r^{\gamma}\right) \geq \underline{\alpha}\right.$ and the equality holds for some component\}.

Conversely, consider $\underline{\alpha} \in \mathbb{N}^{m}$. We only need to prove that $P_{\underline{\alpha}} \subseteq P_{\underline{\alpha}}^{\prime}$. Let $f \in P_{\underline{\alpha}}$ be such that $\underline{\nu}(f)=\beta^{0} \geq \underline{\alpha} . \quad\left\{\left[r_{j}\right]\right\}_{j \in \mathcal{J}}$ generates $\operatorname{gr}_{V} R$, therefore $f+P_{\beta^{0}+\underline{e}}=$ $\sum_{\gamma \in Q^{\prime}} a_{\gamma}[r]^{\gamma}$. Thus $f-\sum_{\gamma \in Q^{\prime}} a_{\gamma} r^{\gamma} \in P_{\underline{\beta}^{0}+\underline{e}}$ and as a consequence, $f+f_{0} \in P_{\beta^{0}+\underline{e}}$ for some $f_{0} \in P_{\beta^{0}}^{\prime}$. Analogously, we can get $\beta^{1} \in \mathbb{N}^{m}$, such that $\beta^{1}>\beta^{0}$ and $f+f_{0} \in P_{\beta^{1}}^{\prime}+P_{\beta^{1}+\underline{e}}$. Iterating, it holds that

$$
f \in \bigcap_{j=0}^{\infty}\left(P_{\beta^{0}}^{\prime}+P_{\beta^{j}+\underline{e}}\right),
$$


where $\beta^{0}<\beta^{1}<\cdots<\beta^{i}<\cdots$ are elements in $\mathbb{N}^{m}$. Assume that there exists a finite generating sequence for the valuation $\nu_{1}$. Then, the equality (6.1) for the set $\left\{\nu_{1}\right\}$ proves that $P_{\alpha_{1}}^{\left\{\nu_{1}\right\}} \subseteq m^{\mu_{\alpha}}$ and that $\underline{\alpha}^{\prime}>\underline{\alpha}$ implies $\mu_{\underline{\alpha}^{\prime}}>\mu_{\underline{\alpha}}$, whenever $\mu_{\underline{\alpha}}:=\min \left\{\sum_{j=1}^{s} \gamma_{j} \mid \gamma \in Q_{\underline{\alpha}}\right\}$. Thus, $P_{\beta^{j}+\underline{e}} \subseteq P_{\beta_{1}^{j}+1}^{\left\{\nu_{1}\right\}} \subseteq m^{\mu_{\beta_{1}^{j}}}$. So

$$
\bigcap_{j=0}^{\infty}\left(P_{\beta^{0}}^{\prime}+P_{\beta^{j}+\underline{e}}\right) \subseteq \bigcap_{j=0}^{\infty}\left(P_{\beta^{0}}^{\prime}+m^{j}\right) .
$$

Furthermore, the opposite inclusion also happens because $R$ is a Noetherian domain and $P_{\beta}$ an $m$-primary ideal. Finally considering the ideal of the quotient ring $R / P_{\beta^{0}}^{\prime}, m+P_{\beta^{0}}^{\prime}=\bar{m}$, one gets

$$
\bigcap_{j=0}^{\infty}\left(P_{\beta^{0}}^{\prime}+P_{\beta^{j}+\underline{e}}\right)=\bigcap_{j=0}^{\infty} \bar{m}^{j}=P_{\beta^{0}}^{\prime} .
$$

Hence $f \in P_{\underline{\alpha}}^{\prime}$ because $f \in P_{\beta^{0}}^{\prime}$.

REMARK 6.5. Notice that if $r \in m$ and $\underline{\alpha}=\underline{\nu}(r)$, then $r \in P_{\beta}^{V}$ for any $\underline{\beta} \leq \underline{\alpha}$. Denote $[r]_{\underline{\beta}}:=r+P_{\underline{\beta}+\underline{e}}$. So, $[r]_{\underline{\beta}} \neq 0$ if, and only if, $\underline{\beta}+\underline{e} \not \leq \underline{\alpha}$. That is $[r]$ in Proposition 6.4 means $[r]:=\left\{[r]_{\beta} \mid \underline{\beta} \leq \underline{\alpha}\right.$ and $\beta+\underline{e} \underline{\underline{\alpha}} \underline{\underline{\alpha}}$, although for simplicity's sake, in the above proof, it means $[\bar{r}]_{\underline{\beta}}$ for suitable $\underline{\beta}$.

The main result for the Poincaré series of these families $V$ is the following (see [12]).

THEOREM 6.6. Let $V=\left\{v_{i}\right\}_{i=1}^{m}$ be a family of monomial valuations (of $K$ centered at $R$ ) with respect to a finite system $\Lambda=\left\{r_{j}\right\}_{j=1}^{n}$ of generators of $m$. Then, the multi-graded Poincaré series of $\operatorname{gr}_{V} R, H_{\mathrm{gr}_{V}} R(\underline{t})$, is a rational function. Moreover, a denominator of $H_{\mathrm{gr}_{V}} R(\underline{t})$ is given by

$$
\prod\left(1-\left(t_{1}^{\delta_{1}}\right)^{\alpha_{j 1}}\left(t_{2}^{\delta_{2}}\right)^{\alpha_{j 2}} \cdots\left(t_{m}^{\delta_{m}}\right)^{\alpha_{j m}}\right)
$$

where we have written $\nu_{i}\left(r_{j}\right)=\alpha_{j i},(1 \leq i \leq m ; 1 \leq j \leq n)$ and the product runs over all expressions $\left(1-\left(t_{1}^{\delta_{1}}\right)^{\alpha_{j 1}}\left(t_{2}^{\delta_{2}}\right)^{\alpha_{j 2}} \cdots\left(t_{m}^{\delta_{m}}\right)^{\alpha_{j m}}\right)$ with $1 \leq j \leq n, \delta_{i} \in\{0,1\}$ $(1 \leq i \leq m)$ and not all the $\delta_{i}$ 's are equal to 0 . where

Theorem 6.6 can be proved taking into account that $H_{\mathrm{gr}_{V} R}(\underline{t})=\sum_{i=0}^{m-1} h_{i}$,

$$
h_{i}=\sum_{\underline{\alpha} \in \mathbb{N}^{m}} \operatorname{dim}_{k}\left(P_{\underline{\alpha}+\underline{e}_{\leq i}}^{V} / P_{\underline{\alpha}+\underline{e}_{\leq i+1}}^{V}\right) \underline{t}^{\underline{\alpha}}
$$

is the Poincaré series of the graded algebra $\oplus_{\underline{\alpha} \in \mathbb{N}^{m}} P_{\underline{\alpha}^{\prime} \underline{e}_{\leq i}} / P_{\underline{\alpha}^{\prime}+\underline{e}_{\leq i+1}}$. Interesting families of valuations satisfy the requirements of Theorem 6.6 as one can see in the following result.

THEOREM 6.7. 12 Let $R$ be either a two-dimensional regular local ring or the local ring of a rational surface singularity. Let $V=\left\{\nu_{i}\right\}_{i=1}^{m}$ be a family of divisorial valuations of $K$ centered at $R$. Then $V$ has a finite generating set.

Proof. Let $\pi: Y \rightarrow \operatorname{Spec} R=X$ be a resolution of singularities of $X$ such that if $\left\{E_{j}\right\}_{j=1}^{q}$ are the irreducible components of the exceptional divisor of $\pi$, then the center of each valuation $\nu_{i}, i \leq i \leq m$, is some of the $E_{j}$ ' that we denote by $E_{i}$ and $\pi$ is minimal with that property. Let $E^{\diamond}:=\bigoplus_{j=1}^{q} \mathbb{Z} E_{j}$ be the group of the divisors 
$\left\{E_{j}\right\}_{j=1}^{q}$ and $T$ the set of $m$-primary complete ideals $\mathcal{I} \subset R$ such that $\mathcal{I O}_{Y}$ is an invertible sheaf. For those ideals $\mathcal{I}$, denote by $D_{\mathcal{I}} \in E^{\diamond}$ the unique exceptional divisor such that $\mathcal{I} \mathcal{O}_{Y}=\mathcal{O}_{Y}\left(-D_{\mathcal{I}}\right) . T$ is a finitely generated semigroup because $T$ is isomorphic to the sub-semigroup of $E^{\diamond}$ of lattice points $D$ which are inside the rational polyhedral in $E^{\diamond} \otimes_{\mathbb{Z}} \mathbb{Q}$ given by the constrains $(-D) E_{j} \geq 0$ for all $j$.

Consider generators $\left\{\mathcal{I}_{l}\right\}_{l=1}^{t}$ of the semigroup $T$. For each $l$, pick a set of generators of $\mathcal{I}_{l}$ and denote by $\Lambda=\left\{r_{s}\right\}_{s=1}^{n}$ the set union of the above chosen sets of generators for all integers $l$. $\Lambda$ is a generating set of the set $V$ and to prove it we only need to check that every ideal $P_{\alpha}^{V}$ is generated by the monomials in the $r_{s}$ 's. Consider the divisor $D_{\alpha}^{\prime}=\sum_{i=1}^{m} \alpha_{i} E_{i}$ and apply the Laufer algorithm to find another divisor $D_{\underline{\alpha}} \in E^{\diamond}$ with $\left(-D_{\underline{\alpha}}\right) E_{j} \geq 0$ for all $j$ and such that

$$
P_{\underline{\alpha}}^{V}=\pi_{*}\left(\mathcal{O}_{Y}\left(-D_{\underline{\alpha}}^{\prime}\right)\right)=\pi_{*}\left(\mathcal{O}_{Y}\left(-D_{\underline{\alpha}}\right)\right) \text {. }
$$

As a consequence, for suitable nonnegative integers $a_{l}, P_{\alpha}^{V}=\prod_{l=1}^{t} \mathcal{I}_{l}^{a_{l}}$ and since each ideal $I_{j}$ is spanned by monomials in the set $\left\{r_{s}\right\}_{s=1}^{n}, P_{\underline{\alpha}}^{V}$ is also generated by monomials in the $r_{s}$ 's.

\subsection{Families of plane divisorial valuations.}

6.2.1. Semigroup of values and graded algebra. Along this section $V=\left\{\nu_{i}\right\}_{i=1}^{m}$ will be a finite family of plane divisorial valuations and we shall assume that $R$ is complete; we know that its Poincaré series is a rational function and our goal is to compute this series and to give more information about its value semigroup. We also relate these data with the corresponding data for the close and rather studied families of valuations attached to plane curve singularities $[\mathbf{6}, \mathbf{8}$.

The semigroup of values of $V$ is defined to be the additive sub-semigroup $S_{V}$ of $\mathbb{Z}^{m}$ given by

$$
S_{V}=\left\{\underline{\nu}(f):=\left(\nu_{1}(f), \ldots, \nu_{m}(f) \mid f \in R \backslash\{0\}\right\} .\right.
$$

We also need to consider the minimal resolution of $V$, which is a modification $\pi: X \rightarrow \operatorname{Spec} R$ such that $\nu_{i}$ is the $E_{a(i)}$-valuation for an irreducible component of the exceptional divisor $E$ given by $\pi, 1 \leq i \leq m$, and $\pi$ is minimal with this property.

On the other hand, let $C=\bigcup_{i=1}^{m} C_{i}$ be a reduced germ of curve, with irreducible components $C_{1}, \ldots, C_{m}$, defined by an element $f \in R$, and denote by $R /(f)^{*}$ the set of nonzero divisors of the ring $\mathcal{O}_{C}:=R /(f)$. The semigroup of values $S_{C}$ of $C$ is the additive sub-semigroup of $\mathbb{Z}^{m}$ given by

$$
S_{C}:=\left\{\underline{v}(g)=\left(v_{1}(g), \ldots, v_{m}(g)\right) \mid g \in R /(f)^{*}\right\},
$$

where each $v_{i}$ is the valuation corresponding to $C_{i}$. The dual graph of $C$, denoted by $\mathcal{G}$, is the dual graph of its minimal embedded resolution, attaching an arrow, for each irreducible component $C_{i}$ of $C$, to the vertex corresponding to the exceptional component which meets the strict transform on $X$ of $C_{i}$. Here, we can also consider the valuation ideals $P_{\underline{\alpha}}^{C}:=\left\{g \in \mathcal{O}_{C} \mid \underline{v}(g) \geq \underline{\alpha}\right\} \cup\{0\}$ and the corresponding graded algebra

$$
\operatorname{gr} \mathcal{O}_{C}:=\bigoplus_{\underline{\alpha} \in\left(\mathbb{Z}_{\geq 0}\right)^{m}} \frac{P_{\underline{\alpha}}^{C}}{P_{\underline{\alpha}+\underline{e}}^{C}},
$$


and we shall say that $\Lambda \subset m$ is a generating sequence of $C$ whenever the ideals $P_{\underline{\alpha}}^{C}$ are generated by the images in $\mathcal{O}_{C}$ of the monomials in $\Lambda$. For convenience, we set $C(\underline{\alpha}):=\frac{P_{\underline{\alpha}}^{C}}{P_{\underline{\alpha}+e}^{C}}$ and $c(\underline{\alpha}):=\operatorname{dim}_{k} C(\underline{\alpha})$.

Let $\mathcal{G}$ denote the dual graph (defined as in the case of a unique valuation) attached to $V$. For each vertex $a \in \mathcal{G}, Q_{a}$ denotes some irreducible element of $m$ such that the strict transform of the associated germ of curve $C_{Q_{a}}$ on $X$ is smooth and meets $E_{a}$ transversely. A general curve $C$ of $V$ is a reduced plane curve with $m$ branches defined by $m$ different equations given by general elements of each valuation $\nu_{i}$. An element $\underline{\alpha} \in S_{V}$ is said to be indecomposable if we cannot write $\underline{\alpha}=\underline{\beta}+\underline{\gamma}$ with $\underline{\beta}, \underline{\gamma} \in S_{V} \backslash\{0\}$. In both cases $(V$ and $C) \mathcal{G}$ is a tree, 1 denotes the vertex corresponding to the first exceptional divisor, $\mathcal{E}$ the set of dead ends (those which have only one adjacent vertex, where, to count adjacency, arrows must also be taken into account) and $[a, b]$ the path joining the vertices $a$ and $b$ in $\mathcal{G}$. In the case of plane valuations, for $1 \leq i \leq m, a(i)$ denotes the vertex of $\mathcal{G}$ corresponding to the defining divisor of $\nu_{i}$ and otherwise the $a(i)$ 's are the vertices with arrow of the dual graph of $C$; finally, for each vertex $r \in \mathcal{E}$, denote by $b_{r}$ the nearest vertex to $r$ in $\Omega=\bigcup_{i=1}^{m}[\mathbf{1}, a(i)]$. Define

$$
\mathcal{H}:=\{\mathbf{1}\} \cup \mathcal{E} \cup\left(\Omega \backslash\left\{\Gamma \cup\left\{b_{r} \mid r \in \mathcal{E}\right\}\right\}\right),
$$

where $\Gamma=\bigcap_{i=1}^{r}[\mathbf{1}, \alpha(i)]$. The following result, which holds for a a reduced germ of curve $C$ as above, is proved in [6].

TheOrem 6.8. The set of indecomposable elements of the semigroup $S_{C}$ is

$$
\left\{\underline{v}\left(Q_{a}\right) \mid a \in \mathcal{H}\right\} \cup\left\{\underline{v}\left(Q_{a(i)}\right)+(0, \ldots, 0, l, 0, \ldots, 0) \mid i=1, \ldots, m \quad l \geq 1\right\} .
$$

This theorem allows us to prove the following one concerning the set $V$ [19].

THEOREM 6.9. The set of indecomposable elements of the semigroup of values $S_{V}$ is the set $\left\{\underline{\nu}\left(Q_{a}\right) \mid a \in \mathcal{H}\right\}$. In particular, $S_{V}$ is finitely generated.

Proof. If $C=\bigcup_{i=1}^{m} C_{i}$ is any general curve of $V$, then $S_{V} \subseteq S_{C}$, therefore, by Theorem 6.8, the elements in the set $\left\{\underline{\nu}\left(Q_{a}\right) \mid a \in \mathcal{H}\right\}$ are indecomposable. Conversely, given $h \in R$ such that $\underline{\nu}(h)$ is indecomposable in $S_{V}$, choose a general curve $C$ of $V$ such that the strict transforms of $C$ and $C_{h}$ by the minimal resolution of $V$ do not intersect. Consider the map $\underline{v}$ given by the valuations associated with $C$, then $\underline{\nu}(h)=\underline{v}(h)$ and $\underline{\nu}\left(Q_{a}\right)=\underline{v}\left(Q_{a}\right)$ for any vertex $a . h$ must be irreducible and by the proof of Theorem 6.8, $\underline{v}(h)$ decomposes in $S_{C}$ as sum of elements $\underline{v}\left(Q_{b}\right)$ with $b \in \mathcal{H}$, which proves that $\underline{\nu}(h)=\underline{\nu}\left(Q_{a}\right)$ for some $a \in \mathcal{H}$.

Now we can say that the semigroup $S_{V}$ has no conductor whenever $m>1$, that is, there is no element $\delta \in S_{V}$ such that $\delta+\mathbb{Z}_{\geq 0}^{m} \subseteq S_{V}$. However, the semigroup of values of a curve with $m$ branches does have a conductor $\delta$ and thus, it cannot be finitely generated if $m>1$. In particular, if $C$ is any general curve of $V, S_{V} \neq S_{C}$ when $m>1$ (recall that $S_{V}=S_{C}$ when $m=1$ ).

In the sequel, we shall use the following notations: for $J \subset I:=\{1,2, \ldots, m\}$, $\underline{e}_{J}$ is the element of $\mathbb{Z}^{m}$ whose $j$ th component is 1 whenever $j \in J$ and 0 otherwise, $D(\underline{\alpha})=P_{\underline{\alpha}}^{V} / P_{\underline{\alpha}+\underline{e}}^{V}, D_{i}(\underline{\alpha})=P_{\underline{\alpha}}^{V} / P_{\underline{\alpha}+\underline{e}_{i}}^{V}, d(\underline{\alpha})=\operatorname{dim}_{k} D(\underline{\alpha})$ and $d_{i}(\underline{\alpha})=\operatorname{dim}_{k} D_{i}(\underline{\alpha})$ when $1 \leq i \leq m$. Also, we shall write $B^{i}=\underline{\nu}\left(Q_{a(i)}\right)$. We summarize in the 
following propositions some results concerning those vector spaces and dimensions. As we shall see, interesting results can be deduced from them. Firstly, we shall give a theorem containing an explicit description of the semigroup $S_{V}$ (see [19] for proofs).

Proposition 6.10. With the above notations assume $i \in I$ and $\underline{\alpha} \in \mathbb{Z}^{m}$, then the following properties hold:

(1) The natural homomorphism $D\left(B^{i}\right) \rightarrow D_{i}\left(B^{i}\right)$ is an isomorphism.

(2) $d_{i}(\underline{\alpha}) \geq 2$ if and only if $d_{i}\left(\underline{\alpha}-B^{i}\right) \geq 1$.

(3) Assume that $d_{i}(\underline{\alpha}) \neq 0$ then $d_{i}\left(\underline{\alpha}+B^{i}\right)=1+d_{i}(\underline{\alpha})$.

Proposition 6.11. In this proposition, we assume $i \in I$ and $\underline{\alpha} \in S_{V}$, then

(1) $d_{i}(\underline{\alpha}) \geq 2$ if and only if $\underline{\alpha}-B^{i} \in S_{V}$.

(2) If $I \ni j \neq i$, then $d_{i}\left(\underline{\alpha}+B^{j}\right)=d_{i}(\underline{\alpha})$.

TheOREM 6.12. Let $\underline{\alpha} \in S_{V}$, then there exist unique nonnegative integers $z_{i}$, $1 \leq i \leq m$, and a unique value $\underline{\beta} \in S_{V}$ such that

- $\underline{\alpha}=\underline{\beta}+\sum_{i=1}^{m} z_{i} B^{i}$.

- $d_{i}(\underline{\beta})^{-}=1$ for every $i$.

Each value $z_{i}$ satisfies the following equality $z_{i}=\max \left\{l \in \mathbb{Z}, l \geq 0 \mid \underline{\alpha}-l B^{i} \in\right.$ $\left.S_{V}\right\}=d_{i}(\underline{\alpha})-1$.

Proof. First, let us prove that there exist the values $z_{i}$ and $\beta$. Indeed, define $z_{i}=\max \left\{l \in \mathbb{Z}, l \geq 0 \mid \underline{\alpha}-l B^{i} \in S_{V}\right\}$ and $\beta=\underline{\alpha}-\sum_{i=1}^{m} z_{i} \bar{B}^{i}$. It suffices to show that $\underline{\alpha}-B^{i} \in S_{V}$ and $\underline{\alpha}-B^{j} \in S_{V}$ imply $\underline{\alpha}-B^{i}-B^{j} \in S_{V}$. Indeed, propositions 6.10 and 6.11 allows us to state that $d_{j}\left(\underline{\alpha}-B^{i}\right)=d_{j}(\underline{\alpha}) \geq 2$ and hence that $\alpha-B^{i}-B^{j} \in S_{V}$. To finish we prove uniqueness: Proposition 6.11 proves $1=\bar{d}_{i}(\underline{\beta})=d_{i}\left(\underline{\alpha}-z_{i} B^{i}\right)=d_{i}(\underline{\alpha})-z_{i}$, and by Proposition 6.11 it holds that $\beta-B^{i} \notin S_{V}$, thus $z_{i}=\max \left\{l \in \mathbb{Z}, l \geq 0 \mid \underline{\alpha}-l B^{i} \in S_{V}\right\}=d_{i}(\underline{\alpha})-1$.

Proposition 6.4 proves that $V$ has a finite minimal generating sequence. Next result, proved in [19], shows how minimal generating sequences for $V$ and for general curves $C$ of $V$ are. As above $\mathcal{G}$ denotes the dual graph attached either to $V$ or to $C$, consider $f_{i} \in R$ which gives an equation for $C_{i}$ and fix an element $Q_{r} \in R$ for each $r \in \mathcal{E}$. Set

$$
\Lambda_{\mathcal{E}}:=\left\{Q_{r} \mid r \in \mathcal{E}\right\} \text { and } \Lambda_{\overline{\mathcal{E}}}:=\left\{Q_{r} \mid r \in \mathcal{E}\right\} \cup\left\{f_{i}\right\}_{i=1}^{m},
$$

where we do not include $f=f_{1}$ whenever $m=1$, then,

THEOREM 6.13. The set $\Lambda_{\mathcal{E}}\left(\Lambda_{\overline{\mathcal{E}}}\right.$, respectively) is a minimal generating sequence of $V$ ( $C$, respectively). Moreover, any minimal generating sequence for $V$ and $C$ is of the described form.

6.2.2. Poincaré series. In this subsection, we shall introduce a Poincaré series for finite families $V$ of plane divisorial valuations (and also for general elements attached to those families) that contains the same information provided for the Poincaré series attached to their corresponding graded algebras. In this form it is easier to compute those series. Assume $m>1$ and set $\mathcal{L}:=\mathbb{Z}\left[\left[t_{1}, t_{1}^{-1}, \ldots, t_{m}, t_{m}^{-1}\right]\right]$. As above $\underline{t}=\left(t_{1}, \ldots, t_{m}\right)$ and $\underline{t} \underline{\alpha}:=t_{1}^{\alpha_{1}} \cdots t_{m}^{\alpha_{m}}$, for $\underline{\alpha}=\left(\alpha_{1}, \ldots, \alpha_{m}\right) \in \mathbb{Z}^{m}$. Clearly $\mathcal{L}$ is a $\mathbb{Z}\left[t_{1}, \ldots, t_{r}\right]$-module and a $\mathbb{Z}\left[t_{1}, t_{1}^{-1}, \ldots, t_{r}, t_{r}^{-1}\right]$-module. 
For a reduced plane curve $C$ with $m$ branches, the formal Laurent series $L_{C}(\underline{t}):=\sum_{\underline{\alpha} \in \mathbb{Z}^{m}} c(\underline{\alpha}) \underline{t} \underline{\alpha} \in \mathcal{L}$ was introduced in $\underline{\mathbf{8}}$. There, the authors showed that $P_{C}^{\prime}(\underline{t})=L_{C}(\underline{t}) \prod_{i=1}^{m}\left(t_{i}-1\right)$ is a polynomial that is divisible by $t_{1} \cdots t_{m}-1$. The Poincaré series for the curve $C$ is defined as the polynomial with integer coefficients $P_{C}(\underline{t})=P_{C}^{\prime}(\underline{t}) /\left(t_{1} \cdots t_{m}-1\right)$. In our case, a finite family $V$ of plane divisorial valuations, we define

$$
L_{V}\left(t_{1}, \ldots, t_{m}\right)=\sum_{\underline{\alpha} \in \mathbb{Z}^{m}} d(\underline{\alpha}) \underline{t}^{\underline{\alpha}} \in \mathcal{L} .
$$

The series $L_{V}$ is a Laurent series, but, since $d(\underline{\alpha})$ can be positive even if $\underline{\alpha}$ have some negative component $\alpha_{i}$, it is not a power series. It can be proved [19] that $P_{V}^{\prime}(\underline{t}):=L_{V}(\underline{t}) \prod_{i=1}^{m}\left(t_{i}-1\right) \in \mathbb{Z}\left[\left[t_{1}, \ldots, t_{m}\right]\right]$. We define the Poincaré series of $V$ as

$$
P_{V}\left(t_{1} \ldots, t_{m}\right)=\frac{P_{V}^{\prime}\left(t_{1}, \ldots, t_{m}\right)}{t_{1} \cdots t_{m}-1},
$$

which is also a formal power series. Write $\widetilde{P}_{V}^{\prime}(\underline{t})=H_{\operatorname{gr}_{V} R}(\underline{t}) \prod_{i=1}^{m}\left(t_{i}-1\right)$, then

$$
\widetilde{P}_{V}^{\prime}\left(t_{1}, \ldots, t_{m}\right)=\left.\sum_{J \subset I}(-1)^{\operatorname{card}(J)} P_{V}^{\prime}(\underline{t})\right|_{\left\{t_{i}=1 \text { for } i \in J\right\}} .
$$

So one can compute $H_{\mathrm{gr}_{V}} R(\underline{t})$ from $P_{V}^{\prime}(\underline{t}) . H_{\mathrm{gr}_{V}} R(\underline{t})$ determines the series $L_{V}(\underline{t})$ since $d(\underline{\alpha})=d\left(\max \left(\alpha_{1}, 0\right), \ldots, \max \left(\alpha_{m}, 0\right)\right)$ for $\alpha \not \leq-1=(-1, \ldots,-1)$ and $d(\underline{\alpha})=$ 0 for $\underline{\alpha} \leq-1$. The next result shows the relation between the Poincaré series of $V$ and a general curve for it.

Theorem 6.14. 19] Let $V=\left\{\nu_{i}\right\}_{i=1}^{m}$ be a finite family of plane divisorial valuations and $C$ a general curve for $V$, then the following equality holds.

$$
P_{V}\left(t_{1}, \ldots, t_{m}\right)=\frac{P_{C}\left(t_{1}, \ldots, t_{m}\right)}{\prod_{i=1}^{r}\left(1-\underline{t}^{B^{i}}\right)} .
$$

For a vertex $a$ of the dual graph $\mathcal{G}$ of a set of valuations $V$ as above, we denote by $\dot{E}_{a}=E_{a} \backslash\left(\overline{E-E_{a}}\right)$ the smooth part of an irreducible component $E_{a}$ in the exceptional divisor $E$ of the minimal resolution of $V$ and by $\chi\left(\dot{E}_{a}\right)$ its Euler characteristic. In addition, set $\underline{\nu}^{a}:=\underline{\nu}\left(Q_{a}\right)$. When the field $k$ is the field of complex numbers and $R=\mathcal{O}_{\mathbb{C}^{2}, O}$ is the local ring of germs of holomorphic functions at the origin of the complex plane, the following formula of A'Campo's type $\mathbf{1}$ holds. (See [19, 20]).

THEOREM 6.15 .

$$
P_{V}\left(t_{1}, \ldots, t_{m}\right)=\prod_{E_{a} \subset E}\left(1-\underline{t}^{\nu^{a}}\right)^{-\chi\left(\dot{E}_{a}\right)} .
$$

6.2.3. Families of plane valuations. The Poincaré series for families of plane valuations of the fraction field of $R=\mathcal{O}_{\mathbb{C}^{2}, O}$, centered at $R$, has been treated in 10. Consider a finite family $V=\left\{\nu_{1}, \ldots, \nu_{m}\right\}$ of plane valuations, denote by $S_{i}$ the value semigroup of $\nu_{i}$, set $S:=S_{1} \times \cdots \times S_{m}$ and, for any $\underline{\alpha} \in S$, define $P_{\alpha}^{V}$ as above and $P_{\underline{\alpha}+}^{V}:=\{f \in R \mid \underline{\nu}(f)>\underline{\alpha}\}$. The usual definition of Poincaré series has no sense for any type of family $V$, so the authors define the Poincaré series of $V$, 
$P_{V}$, by means of the following expression that coincides with the usual definition whenever the valuations are integer valuated:

$$
P_{V}\left(t_{1}, \ldots, t_{m}\right)=\sum_{\underline{\alpha} \in S}\left(\sum_{J \subseteq I}(-1)^{\operatorname{card}(J)} \operatorname{dim} \frac{P_{\underline{\alpha}}^{V} \cap P_{\underline{\alpha}_{J}+}^{V_{J}}}{P_{\underline{\alpha}+}^{V}}\right) \underline{t} \underline{\underline{\alpha}},
$$

where for $J \subseteq I$ we have written $V_{J}:=\left\{\nu_{j} \mid j \in J\right\}$ and $\underline{\alpha}_{J}$ is the projection of $\underline{\alpha}$ preserving only the coordinates corresponding to $J$. With the help of projective limits and as in the case of a unique valuation, it is possible to introduce a notion of resolution $\pi: X \rightarrow \mathbb{C}^{2}$ of $V$. Assuming that the valuations of type B-II are exactly $\nu_{i}, 1 \leq i \leq r$, and denoting by $f_{i}$ the last element of a generating sequence of each one of these valuations $\nu_{i}$, it happens the following result, proved in [10] with the help of integration with respect to the Euler characteristic over the projectivization $\mathbb{P} R$ of $R$.

Proposition 6.16. Let $V=\left\{\nu_{i}\right\}_{i=1}^{m}$ be a finite family of plane valuations ordered as we have said. Then the Poincaré series $P_{V}(\underline{t})$ determines the types of the involved valuations, the dual graph of its minimal resolution up to combinatorial equivalence and divisors and sequences of divisors corresponding to valuations. Furthermore, a formula of A'Campo's type for $P_{V}(\underline{t})$ is

$$
P_{V}(\underline{t})=\prod_{E_{a} \subset E}\left(1-\underline{t}^{\underline{\nu^{a}}}\right)^{-\chi\left(\dot{E}_{a}\right)} \times \prod_{i=1}^{r}\left(1-t_{i}^{(1,0)} \prod_{j \neq i} t_{j}^{\nu_{j}\left(f_{i}\right)}\right)^{-1} .
$$

\section{An application: Poincaré series of multiplier ideals of a plane divisorial valuation}

An important tool in singularity theory and birational geometry is the concept of multiplier ideal. Multiplier ideals provide information on the type of singularity attached to an ideal, divisor or metric, see for instance 39. Although this tool is very useful, explicit computations are hard (see [4, 32, 33, 43]). In this section, we summarize the results in $\mathbf{2 6}$ that provide an specific calculation of a Poincaré series containing the essential information corresponding to jumping numbers and dimensions of quotients of consecutive multiplier ideals of the primary simple complete ideal attached to a plane valuation in the complex case. So, with the above notation, assume that $k=\mathbb{C}, \mathbb{C}$ being the field of complex numbers, and let $\nu$ be a plane divisorial valuation of $K$ centered at $R$. It is known 46 that $\nu$ determines (and it is determined by) a simple complete $m$-primary ideal of $R, \mathcal{I}_{\nu}$, and we define jumping numbers and multiplier ideals attached to $\nu$ as the same objects corresponding to $\mathcal{I}_{\nu}$. Consider the blowing-up sequence (4.1) given by $\nu$, being $\pi_{N}: X=X_{N} \rightarrow X_{N-1}$ the last blowing-up, and set $D=\sum_{i=1}^{N} a_{i} E_{i}$ the effective divisor such that $\mathcal{I}_{\nu} \mathcal{O}_{X}=\mathcal{O}_{X}(-D)$, then for any positive rational number $\iota$, the multiplier ideal of $\nu$ and $\iota$ is defined as $\mathcal{J}\left(\nu^{\iota}\right):=\pi_{*} \mathcal{O}_{X}\left(K_{X \mid X_{0}}-\lfloor\iota D\rfloor\right)$, where $K_{X \mid X_{0}}$ is the relative canonical divisor and $\lfloor\cdot\rfloor$ represents the round-down or the integral part of the corresponding divisor. The family of multiplier ideals is totally ordered by inclusion and parameterized by non-negative rational numbers. Furthermore, there is an increasing sequence $\iota_{0}<\iota_{1}<\cdots$ of positive rational numbers, called jumping numbers, such that $\mathcal{J}\left(\nu^{\iota}\right)=\mathcal{J}\left(\nu^{\iota_{l}}\right)$ for $\iota_{l} \leq \iota<\iota_{l+1}$ and $\mathcal{J}\left(\nu^{\iota^{l}+1}\right) \subset \mathcal{J}\left(\nu^{\iota_{l}}\right)$ for each $l \geq 0 ; \iota_{0}$, usually named the log-canonical threshold of $\mathcal{I}_{\nu}$, is the least positive rational number such that $\mathcal{J}\left(\nu^{\iota_{0}}\right) \neq R$. 
The star vertices of the dual graph (labelled with the symbols $s t_{j}$ in Figure 1) will be those whose associated exceptional divisors $E_{s t_{j}}$ meet three distinct prime exceptional divisors. From now on, we shall denote by $g^{*}$ the number of star vertices. Write

$$
\mathcal{H}_{j}:=\left\{\iota(j, p, q, r):=\frac{p}{e_{j-1}}+\frac{q}{\bar{\beta}_{j}}+\frac{r}{e_{j}} \mid \frac{p}{e_{j-1}}+\frac{q}{\bar{\beta}_{j}} \leq \frac{1}{e_{j}} ; p, q \geq 1, r \geq 0\right\}
$$

whenever $1 \leq j \leq g^{*}$, and

$$
\mathcal{H}_{g^{*}+1}:=\left\{\iota\left(g^{*}+1, p, q\right):=\frac{p}{e_{g^{*}}}+\frac{q}{\bar{\beta}_{g^{*}+1}} \mid p, q \geq 1\right\},
$$

$p, q$ and $r$ being integer numbers. In [35], it is proved that the set $\mathcal{H}$ of jumping numbers of $\nu$ can be computed as $\mathcal{H}=\cup_{i=j}^{g^{*}+1} \mathcal{H}_{j}$.

Assume $\iota \in \mathcal{H}$ and $\iota \neq \iota_{0}=\min \mathcal{H}$. We denote by $\iota^{<}$the largest jumping number which is less than $\iota$. By convention we set $\mathcal{J}\left(\nu^{\iota_{0}^{<}}\right)=R$. Nakayama's Lemma proves that, for any $\iota \in \mathcal{H}, \mathcal{J}\left(\nu^{\iota}\right) / \mathcal{J}\left(\nu^{\iota}\right)$ is a finitely generated $\mathbb{C}$-vector space. Thus, the Poincaré series we referred to will be defined as follows.

Definition 7.1. Let $\nu$ be a plane divisorial valuation. The Poincaré series of multiplier ideals of $\nu$ is defined to be the following fractional power series:

$$
P_{\mathcal{J}, \nu}(t):=\sum_{\iota \in \mathcal{H}} \operatorname{dim}_{\mathbb{C}}\left(\frac{\mathcal{J}\left(\nu^{\iota}\right)}{\mathcal{J}\left(\nu^{\iota}\right)}\right) t^{\iota},
$$

$t$ being an indeterminate.

The main result of this section is to give an explicit computation of the series $P_{\mathcal{J}, \nu}$ which also proves that it is a rational function in certain sense that we shall clarify. The proof is supported in three interesting facts. On the one hand, results and proofs of propositions 6.10 and 6.11, where the family $V$ of involved plane divisorial valuations is given by the $N$ exceptional divisors $E_{i}$ appearing in (4.1), and, on the other hand, the next two propositions. To state the first one, we need the concept introduced in Definition 7.2, where $\pi$ and $D=\sum_{i=1}^{N} a_{i} E_{i}$ are, respectively, the sequence of point blowing-ups and the divisor attached to $\nu$.

DEFINITION 7.2. A candidate jumping number from a prime exceptional divisor $E_{i}$ given by $\pi$ is a positive rational number $\iota$ such that $\iota a_{i}$ is an integer number. We shall say that $E_{i}$ contributes $\iota$ whenever $\iota$ is a candidate jumping number from $E_{i}$ and $\mathcal{J}\left(\nu^{\iota}\right) \subset \pi_{*} \mathcal{O}_{X}\left(-\lfloor\iota D\rfloor+K_{X \mid X_{0}}+E_{i}\right)$.

Proposition 7.3. A jumping number $\iota$ of a plane divisorial valuation $\nu$ belongs to the set $\mathcal{H}_{j}\left(1 \leq j \leq g^{*}+1\right)$ if and only if the prime exceptional divisor $F_{j}$ contributes $\iota$, where $F_{j}$ is defined to be $E_{s t_{j}}$ if $1 \leq j \leq g^{*}$ and $E_{N}$ (the last obtained exceptional divisor) whenever $j=g^{*}+1$.

Jumping numbers and multiplier ideals can also be introduced for analytically irreducible plane curves and for them a similar result to Proposition 7.3 is proved in 48 and 44 . Our proof 26 and the previous ones are independent and use different arguments. Now, we state the second result. 
Proposition 7.4. Let $\iota$ be a jumping number of a plane divisorial valuation $\nu$. Then

$$
\pi_{*} \mathcal{O}_{X}\left(-\lfloor\iota D\rfloor+K_{X \mid X_{0}}+\sum_{l=1}^{s} F_{j_{l}}\right)=\mathcal{J}\left(\nu^{\iota}<\right),
$$

where $\left\{j_{1}, j_{2}, \ldots, j_{s}\right\}$ is the set of indexes $j, 1 \leq j \leq g^{*}+1$, such that $\iota \in \mathcal{H}_{j}$.

We end this paper by stating the mentioned main result.

Theorem 7.5. The Poincaré series $P_{\mathcal{J}, \nu}(t)$ can be expressed as

$$
P_{\mathcal{J}, \nu}(t)=\frac{1}{1-t} \sum_{j=1}^{g^{*}} \sum_{\iota \in \mathcal{H}_{j}, \iota<1} t^{\iota}+\frac{1}{(1-t)^{2}} \sum_{\iota \in \Omega} t^{\iota},
$$

where

$$
\Omega:=\left\{\iota \in \mathcal{H}_{g^{*}+1} \mid \iota \leq 2 \text { and } \iota-1 \notin \mathcal{H}_{g^{*}+1}\right\}
$$

Notice that if one considers the indeterminates $z_{j}=t^{\frac{1}{e_{j-1} \beta_{j}}}$, then $P_{\mathcal{J}, \nu}(t)$ belongs to the field of rational functions $\mathbb{C}\left(z_{1}, z_{2}, \ldots, z_{g^{*}+1}\right)$.

\section{References}

[1] N. A'Campo, La fonction zêta d'une monodromie, Comment. Math. Helv. 50 (1975), 233248. MR0371889(51:8106)

[2] S. S. Abhyankar, Local uniformization on algebraic surfaces over ground field of characteristic $p \neq 0$, Ann. Math. 63 (1956), 491-526. MR0078017 (17:1134d)

[3] S. S. Abhyankar, On the valuations centered in a local domain, Amer. J. Math. 78 (1956), 321-348. MR0082477(18:556b)

[4] N. Budur, Jumping numbers of hyperplane arrangements, Comm. Algebra 38 (2010), 11221136. MR2650395 (2011d:14032)

[5] A. Campillo, "Algebroid curves in positive characteristic", Lecture Notes in Math. 613, Springer-Verlag, 1980. MR584440 (82h:14001)

[6] A. Campillo, F. Delgado, S.M. Gusein-Zade, The extended semigroup of a plane curve singularity, J. London Math. Soc. 60 (1999), 420-430. MR.1724869 (2000m:14002)

[7] A. Campillo, F. Delgado, S.M. Gusein-Zade, The Alexander polynomial of a plane curve singularity and integrals with respect to the Euler characteristic, Int. J. Math. 14 (2003), 47-52. MR1955509 (2004a:14031)

[8] A. Campillo, F. Delgado, S.M. Gusein-Zade, The Alexander polynomial of a plane curve singularity via the ring of functions on it, Duke Math. J. 117 (2003), 125-156. MR.1962784 (2004e:14047)

[9] A. Campillo, F. Delgado, S.M. Gusein-Zade, Poincaré series of a rational surface singularity, Invent. Math. 155 (2004), 41-53. MR2025300 (2004j:14042)

[10] A. Campillo, F. Delgado, S.M. Gusein-Zade, F. Hernando, Poincaré series of collections of plane valuations, Int. J. Math. 21 (2010), 1461-1473. MR.2747738(2012a:13006)

[11] A. Campillo, C. Galindo, On the graded algebra relative to a valuation, Manuscripta Math. 92 (1997), 173-189. MR 1428647(98a:13003)

[12] A. Campillo, C. Galindo, The Poincaré series associated with finitely many monomial valuations, Math. Proc. Camb. Phil. Soc. 134 (2003), 433-443. MR.1981210 (2004d:13022)

[13] V. Cossart, C. Galindo, O. Piltant, Un exemple effectif de gradué non noethérien associé à une valuation divisorielle, Ann. Inst. Fourier 50 (2000), 105-112. MR1762339(2001g:13005)

[14] S.D. Cutkosky, Semigroups of valuations dominating local domains (2008), arxiv:math/0801.0449v1.

[15] S.D. Cutkosky, J. Herzog, A. Reguera, Poincaré series of resolutions of surface singularities, Trans. Amer. Math. Soc. 356 (2004), 1833-1874. MR2031043 (2005a:14015)

[16] S.D. Cutkosky, V. Srinivas, On a problem of Zariski on dimensions of linear systems, Ann. Math. 137 (1993), 531-559. MR1217347 (94g:14001) 
[17] S.D. Cutkosky, B. Teissier, Semigroups of valuations on local rings II (2008), to appear in Amer. J. Math. MR2732345(2011k:13005)

[18] F. Delgado, C. Galindo, A. Nuñez, Saturation for valuations on two-dimensional regular local rings, Math. Z. 234 (2000), 519-550. MR1774096 (2001h:13003)

[19] F. Delgado, C. Galindo, A. Nuñez, Generating sequences and Poincaré series for a finite set of plane divisorial valuations, Adv. Math. 219 (2008), 1632-1655. MR2458149 (2009i:13005)

[20] F. Delgado, S.M. Gusein-Zade, Poincaré series for several divisorial valuations, Proc. Edinb. Math. Soc 46 (2003), 501-509. MR 1998577(2004i:14003)

[21] R. Dedekind, H. Weber, Theorie der algebraischen functionen einer veränderlichen, J. für Math. (1882).

[22] S. Encinas, O. Villamayor, Good points and constructive resolution of singularities, Acta Math. 181 (1998), 109-158. MR 1654779 (99i:14020)

[23] L. Ein, R. Lazarsfeld, K. Smith, Uniform approximation of Abhyankar valuation ideals in smooth function fields, Amer. J. Math. 125 (2003), 409-440. MR1963690 (2003m:13004)

[24] C. Favre, M. Jonsson, "The valuative tree", Lecture Notes in Math. 1853, Springer-Verlag, Berlin, 2004. MR2097722 (2006a:13008)

[25] C. Galindo, On the Poincaré series for a plane divisorial computation, Bull. Belg. Math. Soc. 2 (1995), 65-74. MR 1323928 (96e:13004)

[26] C. Galindo, F. Monserrat, The Poincaré series of multiplier ideals of a simple complete ideal in a local ring of a smooth surface, Adv. Math. 225 (2010), 1046-1068. MR.2671187 (2012a:14039)

[27] C. Galindo, M. Sanchis, Evaluation codes and plane valuations, Des. Codes Crypt. 41 (2006), 199-219. MR2271689(2007h:94086)

[28] P.D. González-Pérez, F. Hernando, Quasi-ordinary singularities, essential divisors and Poincaré series, J. London Math. Soc. 79 (2009), 780-802. MR2506698(2010i:32024)

[29] S. Greco, K. Kiyek, "General elements in complete ideals and valuations centered at a twodimensional regular local ring", in Algebra, Arithmetic, and Geometry, with Applications, Springer (2003), 381-455. MR2037102(2005f:13002)

[30] S.M. Gusein-Zade, F. Delgado, A. Campillo, On the monodromy of a plane curve singularity and the Poincaré series of its ring of functions, Funct. Anal. Appl. 33 (1999), 56-57. MR 1711890 (2000f:32042)

[31] K. Hensel, Uber eine neue begründung der theorie der algebraischen zahlen, Deutsch. Math. 6 (1897).

[32] J. Howald, Multiplier ideals of monomial ideals, Trans. Amer. Math. Soc. 353 (2001), 26652671. MR 1828466 (2002b:14061)

[33] J. Howald, Multiplier ideals for sufficiently general polynomials, arXiv:math/0303203v1.

[34] H. Hironaka, Resolution of singularities of an algebraic variety over a field of characteristic zero, Ann. Math. 79 (1964). MR0199184 (33:7333)

[35] T. Järvilehto, "Jumping numbers of a simple complete ideal in a two-dimensional regular local ring", Ph. D. thesis, University of Helsinky, 2007.

[36] K. Kiyek, J.J. Moyano-Fernández, The Poincaré series of a simple complete ideal of a twodimensional regular local ring, J. Pure Appl. Algebra 213 (2009), 1777-1787. MR.2518176 (2010j:13046)

[37] A. Küronya, A divisorial valuation with irrational volume, J. Algebra 262 (2003), 413—423. MR:1971047 (2004d:13003)

[38] F. V. Kuhlmann, Value groups, residue fields and bad places of algebraic function fields, Trans. Amer. Math. Soc. 356 (2004), 4559-4600. MR2067134(2005d:12010)

[39] R. Lazarsfeld, "Positivity in algebraic geometry. Vol. II", Springer, 2004.

[40] A. Lemahieu, Poincaré series of a toric variety, J. Algebra 315 (2007), 683 - 697. MR.2351887 (2009e:14084)

[41] S. MacLane, A construction for absolute values in polynomial rings, Trans. Amer. Math. Soc. 40 (1936), 363-395. MR 1501879

[42] S. MacLane, O. Schilling, Zero-dimensional brances of rank 1 on algebraic varieties, Ann. Math. 40 (1939), 507-520. MR0000158(1:26c)

[43] M. Mustaţă, Multiplier ideals of hyperplane arrangements, Trans. Amer. Math. Soc. 358 (2006), 5015-5023. MR2231883 (2007d:14007)

[44] D. Naie, Jumping numbers of a unibranch curve on a smooth surface, Manuscripta Math. 128 (2009), 33-49. MR2470185 (2009j:14034) 
[45] A. Nemethi, Poincaré series associated with surface singularities. Proceedings of the international conference "School and workshop on the geometry and topology of singularities" in honor of the 60th birthday of Lê Dung Tráng. Contemporary Math. 474 (2008), 271—297. MR2454352 (2010f:32025)

[46] M. Spivakovsky, Valuations in function fields of surfaces, Amer. J. Math. 112 (1990), 107156. MR1037606 (91c:14037)

[47] B. Teissier, Valuations, deformations and toric geometry. Proceedings of the Saskatoon conference and workshop on valuation theory, Fields Institute Comm. 33 (2003), 361-459. MR2018565 (2005m:14021)

[48] K. Tucker, "Jumping numbers and multiplier ideals on algebraic surfaces" PhD Dissertation, University of Michigan, 2010. MR2736766

[49] O. Villamayor, Constructiveness of Hironaka's resolution, Ann. Sc. Éc. Norm. Sup. 22 (1989), 1-32. MR 985852 (90b:14014)

[50] O. Zariski, Local uniformization on algebraic varieties, Ann. Math. 41 (1940), 852-896. MR.0002864 (2:124a)

[51] O. Zariski, The reduction of singularities of an algebraic surface, Ann. Math. 40 (1939), 639-689. MR0000159 (1:26d)

[52] O. Zariski and P. Samuel, "Commutative Algebra. Vol II", Springer-Verlag, 1960. MR0120249 $(22: 11006)$

Current address: Departamento de Matemáticas \& Instituto Universitario de Matemáticas y Aplicaciones de Castellón (IMAC), Universitat Jaume I. Campus de Riu Sec, 12071 Castellón, Spain.

E-mail address: galindo@mat.uji.es

Current address: Instituto Universitario de Matemática Pura y Aplicada (IUMPA), Universidad Politécnica de Valencia, Camino de Vera s/n, 46022 Valencia, Spain.

E-mail address: framonde@mat.upv.es 